\title{
Treating the symptom or treating the disease in neonatal seizures: a systematic review of the literature
}

\author{
Raffaele Falsaperla ${ }^{1}$, Bruna Scalia ${ }^{1 *} \mathbb{D}$, Andrea Giugno², Piero Pavone ${ }^{3}$, Milena Motta $^{1}$, Martina Caccamo ${ }^{1}$ and \\ Martino Ruggieri ${ }^{4}$
}

\begin{abstract}
Aim: The existing treatment options for neonatal seizures have expanded over the last few decades, but no consensus has been reached regarding the optimal therapeutic protocols. We systematically reviewed the available literature examining neonatal seizure treatments to clarify which drugs are the most effective for the treatment of specific neurologic disorders in newborns.

Method: We reviewed all available, published, literature, identified using PubMed (published between August 1949 and November 2020), that focused on the pharmacological treatment of electroencephalogram (EEG)-confirmed neonatal seizures.

Results: Our search identified 427 articles, of which 67 were included in this review. Current knowledge allowed us to highlight the good clinical and electrographic responses of genetic early-onset epilepsies to sodium channel blockers and the overall good response to levetiracetam, whose administration has also been demonstrated to be safe in both full-term and preterm newborns.

Interpretation: Our work contributes by confirming the limited availability of evidence that can be used to guide the use of anticonvulsants to treat newborns in clinical practice and examining the efficacy and potentially harmful side effects of currently available drugs when used to treat the developing newborn brain; therefore, our work might also serve as a clinical reference for future studies.
\end{abstract}

Keywords: Neonate, Seizure, Treatment, Outcome, Phenobarbital, Levetiracetam

\section{Introduction}

Pediatric patients develop seizures more frequently during the neonatal period than during any other age. The precise incidence of neonatal seizures can be difficult to define and depends on the population being studied and the criteria used for diagnosis. Neonatal seizures have been estimated to occur in up to3-5out of every 1000 births, and preterm newborns are estimated to develop

\footnotetext{
* Correspondence: b.scalia@hotmail.it

${ }^{1}$ Neonatal Intensive Care Unit, A.O.U. San Marco-Policlinico, University of Catania, Via Carlo Azeglio Ciampi, 95121 Catania, Italy

Full list of author information is available at the end of the article
}

seizures more frequently than full- term newborns, with an overall incidence of $10-15$ per1,000 preterm newborns, compared with 3-5 per 1000 full-term newborns and a prevalence of $22.2 \%$ among preterm newborns, compared with $0.5 \%$ among full term newborns $[1,2]$. Moreover, because improved critical care has increased the survival rate following neonatal seizures, long-term neurological sequelae constitute a growing challenge for neonatologists. Greater than $50 \%$ of survivors, especially among preterm newborns, experience considerable disabilities across a range of developmental domains, with cerebral palsy and intellectual disability being the most

C C The Author(s). 2021 Open Access This article is licensed under a Creative Commons Attribution 4.0 International License, which permits use, sharing, adaptation, distribution and reproduction in any medium or format, as long as you give appropriate credit to the original author(s) and the source, provide a link to the Creative Commons licence, and indicate if changes were made. The images or other third party material in this article are included in the article's Creative Commons licence, unless indicated otherwise in a credit line to the material. If material is not included in the article's Creative Commons licence and your intended use is not permitted by statutory regulation or exceeds the permitted use, you will need to obtain permission directly from the copyright holder. To view a copy of this licence, visit http://creativecommons.org/licenses/by/4.0/ The Creative Commons Public Domain Dedication waiver (http://creativecommons.org/publicdomain/zero/1.0/) applies to the data made available in this article, unless otherwise stated in a credit line to the data. 
frequently reported [2-4]. Recent studies have shown a $17.6 \%$ over- all incidence in epilepsy among children with a history of neonatal seizures [5].

Neonatal seizures are often misdiagnosed, resulting in both the under- and overestimation of clinically- diagnosed seizure occurrence, due to electro-clinical dissociation phenomena and because neonatal seizures are often highly focal, with very little spread to other brain regions [6-9]. Therefore, our review focused only on studies that described seizures confirmed by electroencephalography (EEG)- or amplitude-integrated EEG (aEEG).

In addition to making a correct diagnosis, making an early diagnosis of neonatal seizure is fundamental to the administration of proper treatment. Both animal and human studies have demonstrated that recurrent and prolonged seizures are harmful to the developing brain, emphasizing the importance of early seizure recognition and the availability of effective therapy options [10-14].

One of the major challenges facing clinicians who treat neonates with seizures is the lack of effective antiepileptic drugs (AEDs).

Advances on this front have occurred during the last few decades; the anticonvulsant properties of therapeutic hypothermia, for example, have been demonstrated by both preclinical and clinical data. However, a proper, specific, effective, and safe pharmacological treatment for neonatal seizures remains lacking.

Currently, the World Health Organization (WHO) recommends the use of phenobarbital and phenytoin as first-line treatment [15] options for neonatal seizures, despite the low-quality evidence available to support their efficacy and the number of studies highlighting their potential side effects, which include increasing neuronal apoptosis and, consequently, contributing to long-term neurological damage and adverse neurocognitive outcomes [16, 17].

Here, we systematically review the available evidence for the treatment of electrographic and electroclinical neonatal seizures caused by specific neurologic disorders in newborns and evaluate the efficacy of both first-line and add-on anticonvulsants. Data on the populations studied, the seizure etiologies, treatment protocols, and study strengths and limitations were collected $[18,19]$.

\section{Materials and methods}

For this systematic review, we searched the PubMed database using search terms related to neonatal seizures (see below). The search period was from August 1949 to November 2020 (last update 30/11/2020). The only filters applied were publication in the English language and human studies.

A further search of ClinicalTrials.gov was conducted, and a list of ongoing clinical trials is provided.

\section{Search strategy}

The systematic review was conducted following the general principles established by Preferred Reporting Items for Systematic Reviews and Meta-Analyses (PRIS MA) and the Institute of Medicine Standards for Systematic Reviews [20, 21].

Given the lack of robust evidence [randomized clinical trials (RCTs)], we included observational investigations and case reports in our systematic review and focused on evaluating the strengths and methodological limitations of each included study.

The following search strategy was employed: (neonatal seizures treatment) OR (neonatal seizures AND treatment) OR (neonatal seizure antiepileptic drugs) OR (neonatal seizure AND antiepileptic drugs) OR (neonatal seizure phenobarbital) OR (neonatal seizure AND phenobarbital) OR (neonatal seizure phenytoin) OR (neonatal seizure AND phenytoin) OR (neonatal seizure lidocaine) OR (neonatal seizure AND lidocaine) OR (neonatal seizure levetiracetam) OR (neonatal seizure AND levetiracetam) OR (neonatal seizure carbamazepine) OR (neonatal seizure AND carbamazepine) OR (neonatal seizure topiramate) OR (neonatal seizure AND topiramate) OR (neonatal seizure midazolam) OR (neonatal seizure AND midazolam) OR (neonatal seizure valproic acid) OR (neonatal seizure AND valproic acid) OR (neonatal seizure lorazepam) OR (neonatal seizure AND lorazepam) OR (neonatal seizure lacosamide) OR (neonatal seizure AND lacosamide) OR (neonatal seizure lamotrigine) OR (neonatal seizure AND lamotrigine).

\section{Inclusion criteria}

Seizures in full-term infants, only if they occurred within 30 days of birth;

Seizures in preterm infants, only if documented within the postmenstrual age (gestational age plus chronological age, in weeks) of 40 weeks;

Studies describing electro-clinical seizures;

Studies defining a precise etiology underlying seizure onset; and English language studies.

\section{Exclusion criteria}

Studies describing a metabolic, reversible etiology for neonatal seizures, such as metabolic diseases, pyridoxindependent seizures, and electrolytic imbalance disturbances (hypoglycemia and hypocalcemia);

Articles that included EEG and semiology, but described patients who were not in the neonatal period; and Review articles, editorials, letters to the editor, and articles without individual descriptions of seizure semiology and/orEEG. 


\section{Data collection and analysis}

The reviewer screened the title and abstract of each study identified using the above-described search strategy. The same reviewer re-screened the full text of each study that was identified as potentially relevant. Studies meeting any of the pre-specified inclusion criteria were included.

\section{Methodological quality}

Our systematic review was assessed using the "Assessing the Methodological Quality of Systematic Reviews 2" (AMSTAR 2) criteria. According to AMSTAR 2 score, "moderate quality review" result was obtained for this review [22].

\section{Description}

Our PUBMED search for "neonatal seizure treatment" identified 4.829 articles. After reviewing the titles and abstracts of these articles, the authors reviewed the full texts of 427 articles. A total of 67 of these fulfilled the criteria for inclusion in the review (Fig. 1).

Studies were grouped for type of study (RCTs, prospective, retrospective, case reports). In order to ease the access to the large amount of information provided by the literature, of each article we summarized in tables the number of patients included, the etiology beneath the seizures, first-line treatments, add-on therapieswhen available-, and treatment outcomes. Data are listed in the tables below (Tables 1, 2, 3, 4).

\section{Future directions}

A further search on ClinicalTrials.gov for the terms "neonatal seizures" and "neonatal seizures treatment" led to the identification of 5 ongoing clinical trials.

A multicenter, open-label, single-arm study to evaluate the pharmacokinetics, efficacy, and safety of brivaracetam in a cohort of 42 full-term or near-term neonates with repeated electroencephalographic seizures (NCT03325439) is currently recruiting and is estimated to be completed in December 2021.

LEVNEONAT-1 (NCT02229123), is an open-label study evaluating the efficacy and optimal dose of intravenous levetiracetam as a first-line treatment in fullterm or near-term (36-43-week gestational age) newborns with HIE. Patients will be treated with 1 loading dose of $30,40,50$, or $60 \mathrm{mg} / \mathrm{kg}$ and 8 quarterloading maintenance doses for a 3-day treatment. A dose with toxicity not exceeding $10 \%$ and an efficacy greater than $60 \%$ will be considered the optimal dose. Efficacy is defined by the authors as a seizure burden reduction of $80 \%$ after the initial loading dose. The minimal sample expected is 50 participants, with a minimum of 24 patients, although fewer will be used in case of high toxicity.

Another Phase IIb randomized, blinded, controlled study (NCT01720667), which involves 6 different centers in the United States, is currently evaluating the efficacy of levetiracetam for terminating seizures when given as a first-line anticonvulsant in full-term newborns. A large cohort of 280 patients has been enrolled. The efficacy of intravenous levetiracetam (40 to $60 \mathrm{mg} / \mathrm{kg}$ intravenously,

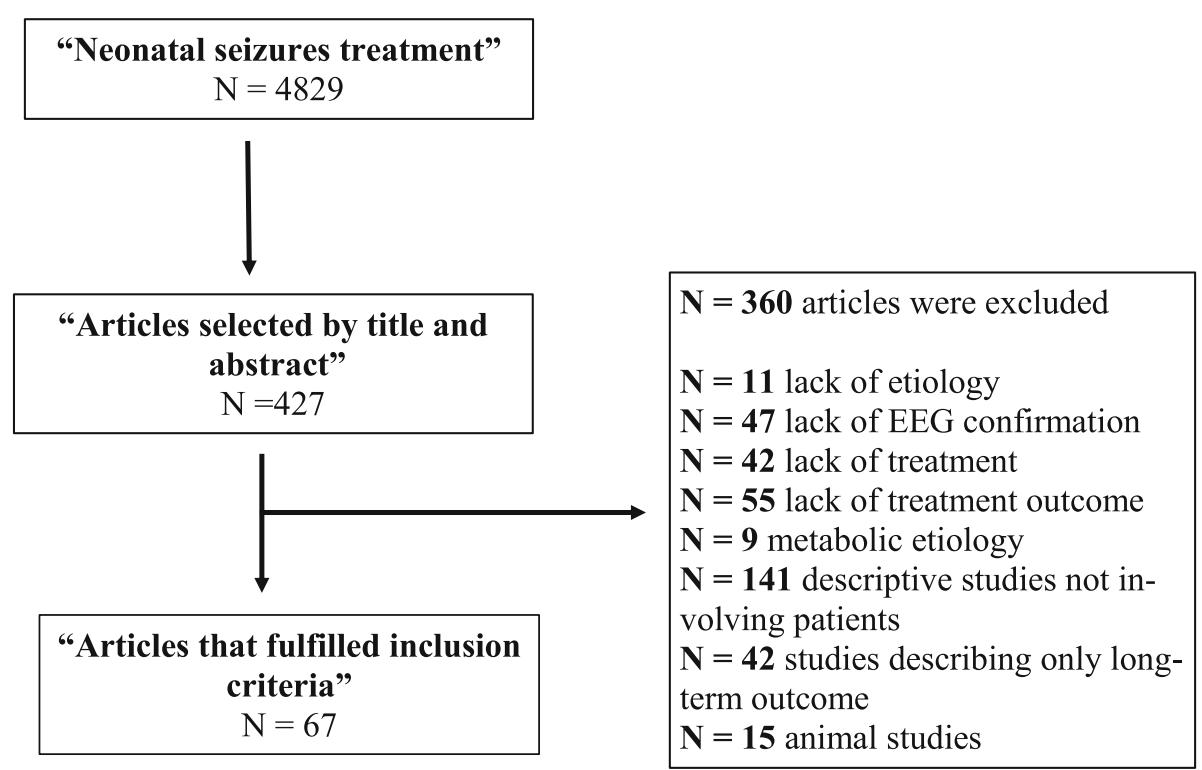

Fig. 1 Search strategy and article selection 
Table 1 Full description of the sources: RCTs

\begin{tabular}{|c|c|c|c|c|c|}
\hline RCTs & Population & Etiology & Treatment & $\begin{array}{l}\text { Add-on } \\
\text { therapy }\end{array}$ & Outcome \\
\hline $\begin{array}{l}\text { Painter } \\
\text { et al. [23] }\end{array}$ & $\begin{array}{l}N=59 ; \text { term } \\
\text { and } \\
\text { preterm }\end{array}$ & $\begin{array}{l}N=49 \mathrm{HIE} \\
N=6 \text { CNS } \\
\text { infections } \\
N=3 \\
\text { cryptogenic }\end{array}$ & $\begin{array}{l}N=30 \mathrm{PHB} \\
N=29 \mathrm{PHE} \\
\text { Dose: N/A }\end{array}$ & $\begin{array}{l}N=15 \mathrm{PHE} \\
\text { as 2nd line } \\
\mathrm{AED} \\
N=13 \mathrm{PHB} \\
\text { as 2nd line } \\
\text { AED }\end{array}$ & $\begin{array}{l}\text { PHB's efficacy: } 43 \% \text {. } \\
\text { PHE's efficacy: } 45 \% \text {. } \\
\text { When combined, efficacy raised to } 57-62 \% \text {. }\end{array}$ \\
\hline $\begin{array}{l}\text { Boylan } \\
\text { et al. [24] }\end{array}$ & $\begin{array}{l}N=22 ; \text { term } \\
\text { and } \\
\text { preterm. }\end{array}$ & $\begin{array}{l}N=13 \mathrm{HIE} \\
N=3 \mathrm{IVH} \\
N=1 \mathrm{BFNE} \\
N=2 \mathrm{IUGR} \\
N=1 \\
\text { premature } \\
N=1 \\
\text { myopathy } \\
N=1 \mathrm{AVM}\end{array}$ & $\begin{array}{l}\mathrm{N}=22 \mathrm{PHB} \\
(20-40 \mathrm{mg} / \mathrm{kg})\end{array}$ & $\begin{array}{l}N=3 \mathrm{MDZ} \\
N=5 \mathrm{LID} \\
N=3 \mathrm{CLZ}\end{array}$ & $\begin{array}{l}50 \% \text { response to } \mathrm{PHB} \text {. } \\
2 / 5 \text { responded to } L I D \text { as } 2 \text { nd line treatment. } \\
\text { No response to } C L Z \text { and } M D Z \text {. }\end{array}$ \\
\hline $\begin{array}{l}\text { Pressler } \\
\text { et al. [25] }\end{array}$ & $\begin{array}{l}N=14 \\
\text { term. }\end{array}$ & HIE & $\begin{array}{l}\text { BMT }(0.05-03 \mathrm{mg} / \mathrm{kg})+ \\
\text { PHB }(10 \mathrm{mg} / \mathrm{kg})\end{array}$ & $\begin{array}{l}N=8 \mathrm{MDZ} \\
N=5 \mathrm{PHE} \\
N=2 \mathrm{LID} \\
N=4 \mathrm{PHB}\end{array}$ & 5/14 had seizure cessation on BMT + PHB. 3/14 had hearing loss. \\
\hline $\begin{array}{l}\text { Falsaperla } \\
\text { et al. [26] }\end{array}$ & $\begin{array}{l}N=30 \\
\text { term. }\end{array}$ & $\begin{array}{l}N=23 \text { HIE } \\
N=3 \\
\text { stroke } \\
N=4 \text { CNS } \\
\text { infection }\end{array}$ & $\begin{array}{l}\mathrm{PHB} \text { ( } 20 \mathrm{mg} / \mathrm{kg} \mathrm{load} \text {, } \\
\text { maintenance } 5 \mathrm{mg} / \mathrm{kg} \text { ) } \\
\text { OR } \\
\text { LEV (load dose } 20 \mathrm{mg} / \mathrm{kg} \text {, } \\
\text { maintenance } 20-40 \mathrm{mg} / \\
\mathrm{kg} \text { ) }\end{array}$ & / & $\begin{array}{l}\text { LEV's administration related to a significantly positive HNNE score } \\
\text { There was no significant improvement in the HNNE score after } \\
\text { one month in the neonates treated with PHB. }\end{array}$ \\
\hline
\end{tabular}

$N$ number of patients; PHB phenobarbital; PHE phenytoin; CNS central nervous system; $A E D$ anti-epileptic drug; N/A not available; HIE hypoxic ischemic encephalopathy; IVH intra-ventricular hemorrhage; BFNE benign familial neonatal epilepsy; IUGR intra-uterine growth restriction; AVM arterio-venous malformation; MDZ midazolam; LID lidocaine; BMT bumetanide; LEV levetiracetam. EEG electroencephalography, HNNE Hammersmith Neonatal Neurological Examination; c.i. continuous infusion; HHNE Hammersmith Neonatal Neurological Examination

followed by a $30 \mathrm{mg} / \mathrm{kg} /$ day maintenance dose) will be compared with that for phenobarbital (20 to $40 \mathrm{mg} / \mathrm{kg}$, followed by $1.5 \mathrm{mg} / \mathrm{kg}$ every $8 \mathrm{~h}$ ).

A multicenter randomized, blinded, controlled, study examining the efficacy of oral levetiracetam as a firstline anticonvulsant in China (NCT02550028) is planning to enroll 100 full-term newborns, with EEG-confirmed seizures, and randomly assign them into either an interventional group treated with intravenous levetiracetam (50 mg/kg, followed by $30 \mathrm{mg} / \mathrm{kg} /$ day) or a control group treated with phenobarbital $(20 \mathrm{mg} / \mathrm{kg})$, with the aim of describing the efficacy of levetiracetam continuous EEG monitoring.

Recently, a randomized, double-blind, parallel-group, phase III study (NCT03602118), with the aim of evaluating the efficacy of phenobarbital sodium injections in participants who have suffered from clinical seizures, has been reported. Because neonatal seizures can have longterm side effects, including death, placebo-controlled studies are not appropriate for this population. The study is designed to demonstrate the effectiveness of phenobarbital for the prevention of subsequent seizures and to demonstrate improved efficacy when used at a higher dose $(40 \mathrm{mg} / \mathrm{kg})$ compared with a lower dose (20 $\mathrm{mg} / \mathrm{kg}$ ). Study participants who experience electrographic or electroclinical seizures that last for $10 \mathrm{~s}$ or longer will be randomized, in a 1:1 fashion, between the 2 treatment arms to receive either a $20 \mathrm{mg} / \mathrm{kg}$ or $40 \mathrm{mg} /$ $\mathrm{kg}$ loading dose of phenobarbital sodium. Participants in the $20 \mathrm{mg} / \mathrm{kg}$ treatment arm in whom seizure activity does not resolve after the first dose will receive phenobarbital in $10 \mathrm{mg} / \mathrm{kg}$ increments (each hour) until seizure activity resolves, up to a maximum dose of $40 \mathrm{mg} / \mathrm{kg}$. If seizure activity still does not resolve, participants will be given a second-line anticonvulsant. Participants in the $40 \mathrm{mg} / \mathrm{kg}$ group in whom seizures do not resolve after the initial loading dose will be given a second-line anticonvulsant. The second line treatment will be determined by the attending physician based, on the patient's clinical history and the seizure's features.

\section{Results}

In the 67 articles included in this review (4 RCT, 11 prospective studies, 27 retrospective studies and 25 case reports), HIE, stroke and genetic channelopathies were the most frequent etiologies of seizures. Despite the number of patients described in this review, performing statistical analyses of the data and providing precise descriptions for how the considered anticonvulsants work was challenging. In an attempt to standardize the results, we grouped all neonates with specific seizure etiologies as though they belonged to a single study. This 
Table 2 Full description of the sources: prospective studies

\begin{tabular}{|c|c|c|c|c|c|}
\hline $\begin{array}{l}\text { Prospective } \\
\text { studies }\end{array}$ & Population & Etiology & Treatment & Add-on therapy & Outcome \\
\hline $\begin{array}{l}\text { Ramantani } \\
\text { et al. } \\
\text { [27] }\end{array}$ & $\begin{array}{l}N=38 \\
\text { term and } \\
\text { preterm. }\end{array}$ & $\begin{array}{l}N=9 \mathrm{HIE} \\
N=16 \mathrm{IVH} \\
N=13 \text { sepsis }\end{array}$ & $\begin{array}{l}N=38 \mathrm{LEV} \\
(10-30 \mathrm{mg} / \\
\mathrm{kg})\end{array}$ & $\begin{array}{l}N=11 \text { PHB as 2nd } \\
\text { line }\end{array}$ & $\begin{array}{l}\text { LEV's efficacy: } 79 \% \text {; } \\
27 / 30(90 \%) \text { remained seizure free at } 4 \text { weeks. }\end{array}$ \\
\hline $\begin{array}{l}\text { Sharpe et al. } \\
{[28]}\end{array}$ & $\begin{array}{l}N=18 \\
\text { term. }\end{array}$ & $\begin{array}{l}N=8 \mathrm{HIE} \\
N=2 \mathrm{IVH} \\
N=1 \text { birth } \\
\text { trauma } \\
N=2 \text { stroke } \\
N=1 \text { brain } \\
\text { malformation } \\
N=4 \mathrm{~N} / \mathrm{A}\end{array}$ & $\begin{array}{l}\text { PHB } \\
(20 \mathrm{mg} / \mathrm{kg})\end{array}$ & $\begin{array}{l}\text { LEV } \\
(20-40 \mathrm{mg} / \mathrm{kg})\end{array}$ & $\begin{array}{l}\text { LEV's efficacy: } 42 \% \text {. } \\
5 \text { of the responders were among the } 12 \text { subjects who received the higher dose } \\
\text { of LEV. }\end{array}$ \\
\hline $\begin{array}{l}\text { Furwentsches } \\
\text { et al. } \\
\text { [29] }\end{array}$ & $\begin{array}{l}N=6 ; \\
\text { term and } \\
\text { preterm }\end{array}$ & $\begin{array}{l}N=1 \mathrm{HIE} \\
N=1 \mathrm{IVH} \\
N=3 \text { brain } \\
\text { malformation } \\
N=1 \mathrm{~N} / \mathrm{A}\end{array}$ & $\begin{array}{l}N=4 \text { oral } \\
L E V \\
(10-50 \mathrm{mg} / \\
\mathrm{kg}) \\
N=2 \mathrm{PHB} \\
(10 \mathrm{mg} / \mathrm{kg})\end{array}$ & $N=2 \mathrm{LEV}$ & $\begin{array}{l}\text { All } 6 \text { patients treated with oral LEV became seizure free within } 6 \text { days. } \\
\text { After } 3 \text { months, five out of six patients were seizure free under monotherapy } \\
\text { with LEV. }\end{array}$ \\
\hline $\begin{array}{l}\text { Falsaperla } \\
\text { et al. } \\
\text { [30] }\end{array}$ & $\begin{array}{l}N=16 \\
\text { late preterm and } \\
\text { term. }\end{array}$ & $\begin{array}{l}N=5 \text { MAS } \\
N=8 \text { RDS } \\
N=3 \text { APD }\end{array}$ & $\begin{array}{l}\text { LEV } \\
(10-40 \mathrm{mg} / \\
\mathrm{kg})\end{array}$ & / & $\begin{array}{l}\text { LEV's efficacy: } 100 \% \text {.Seizure freedom reached from } 24 \mathrm{~h} \text { to } 15 \text { days. EEGs at three } \\
\text { months resulted normal. }\end{array}$ \\
\hline $\begin{array}{l}\text { Boylan et al. } \\
{[31]}\end{array}$ & $N=17$ & $\begin{array}{l}N=7 \mathrm{HIE} \\
N=1 \mathrm{RDS} \\
N=1 \mathrm{IUGR} \\
N=3 \text { sepsis } \\
N=3 \\
\text { prematurity } \\
N=1 \mathrm{NAS} \\
N=1 \\
\text { kernicterus }\end{array}$ & $\begin{array}{l}\mathrm{N}=15 \mathrm{PHB} \\
(20-30 \mathrm{mg} / \\
\mathrm{kg})\end{array}$ & $\begin{array}{l}N=2 \mathrm{PHE} \\
(20 \mathrm{mg} / \mathrm{kg})\end{array}$ & $\begin{array}{l}\text { Full response to PHB in } 6 / 17(35 \%) \text {. } \\
\text { Partial response to PHB in } 6 / 17(35 \%) \text {. } \\
\text { No response in } 2 / 17(12 \%) \text {. }\end{array}$ \\
\hline $\begin{array}{l}\text { Low et al. } \\
\text { [32] }\end{array}$ & $\begin{array}{l}N=19 \text { term or } \\
\text { near term }\end{array}$ & $\begin{array}{l}N=10 \mathrm{HIE} \\
N=6 \text { stroke } \\
N=1 \mathrm{CNS} \\
\text { infection } \\
N=1 \mathrm{BFNE} \\
N=1 \mathrm{~N} / \mathrm{A}\end{array}$ & $\begin{array}{l}\text { PHB } 10-40 \\
\mathrm{mg} / \mathrm{kg} \text { i.v. }\end{array}$ & $\begin{array}{l}\text { PHE or MDZ as } 2 n d \\
\text { line (dose: N/A) }\end{array}$ & $\begin{array}{l}\text { PHB abolished seizurs in } 13 / 19 \text { patients within } 1 \mathrm{~h} \text {. } \\
\text { Only } 3 \text { patients showed permanent reduction. } \\
\text { Loading dose of } 20 \mathrm{mg} / \mathrm{kg} \text { was more effective than } 10 \mathrm{mg} / \mathrm{kg} \text {. }\end{array}$ \\
\hline $\begin{array}{l}\text { Van der Broek } \\
\text { et al. } \\
\text { [33] }\end{array}$ & $\begin{array}{l}N=53 \text { term } \\
\text { newborns }\end{array}$ & HIE & $\begin{array}{l}\mathrm{PHB} 20-40 \\
\mathrm{mg} / \mathrm{kg}\end{array}$ & $\begin{array}{l}\mathrm{MDZ} \text { c.i. } 0.05-0.1 \\
\mathrm{mg} / \mathrm{kg} / \mathrm{h}\end{array}$ & $\begin{array}{l}\text { The observed responsiveness of MDZ add-on therapy after PHB monotherapy } \\
\text { was low (23\%) compared to PHB monotherapy's effectiveness }(66 \%) \text {. }\end{array}$ \\
\hline $\begin{array}{l}\text { Hellstrom- } \\
\text { Westas et al. } \\
{[34]}\end{array}$ & $\begin{array}{l}N=24 \text { both } \\
\text { term and } \\
\text { preterm }\end{array}$ & $\begin{array}{l}N=15 \mathrm{HIE} \\
N=6 \mathrm{IVH} \\
N=2 \mathrm{HIE} \\
N=1 \\
\text { hypoglicemia }\end{array}$ & $\begin{array}{l}N=24 \mathrm{PHB} \\
(10-15 \mathrm{mg} / \\
\mathrm{kg})\end{array}$ & $\begin{array}{l}N=21 \text { DZP as } 2 \mathrm{nd} \\
\text { line } \\
(0.5-2 \mathrm{mg} / \mathrm{kg}) \\
N=24 \mathrm{LID} \text { as } 3 \mathrm{rd} \\
\text { line } \\
(1.6-2.2 \mathrm{mg} / \mathrm{kg})\end{array}$ & $\begin{array}{l}\text { LID's introduction conducted to seizure cessation in 15/24 patients. } \\
2 \text { of them developed bradycardia and acidosis. }\end{array}$ \\
\hline $\begin{array}{l}\text { Maytal et al. } \\
{[35]}\end{array}$ & $N=7$ & $\begin{array}{l}\mathrm{N}=4 \mathrm{HIE} \\
N=2 \mathrm{IVH} \\
N=1 \mathrm{CNS} \\
\text { infections }\end{array}$ & $\begin{array}{l}N=7 \text { PHB } \\
(20-40 \mathrm{mg} / \\
\mathrm{kg})\end{array}$ & $\begin{array}{l}N=7 \mathrm{LRZ} \\
(0.05 \mathrm{mg} / \mathrm{kg} \text { i.v. })\end{array}$ & $\begin{array}{l}\text { After PHB's failure, } 6 / 7 \text { patients had complete cessation of seizures within } 3 \mathrm{~min} \text {. } \\
4 / 6 \text { remained seizure-free on follow-up. } \\
\text { No side-effects were reported. }\end{array}$ \\
\hline Glass et al. [36] & $N=92$ & $\begin{array}{l}N=30 \mathrm{HIE} \\
N=25 \mathrm{IVH}\end{array}$ & $\begin{array}{l}N=\mathrm{N} / \mathrm{A} \\
\mathrm{PHB} \\
(20 \mathrm{mg} / \mathrm{kg}) \\
N=\mathrm{N} / \mathrm{A} \\
\text { LEV } \\
\text { (dose: N/A) } \\
N=\mathrm{N} / \mathrm{A} \\
\mathrm{PHE} \\
\text { (dose: N/A) }\end{array}$ & N/A & $\begin{array}{l}64 \% \text { had seizures that persisted after loading doses of PHB, } 58 \% \text { after LEV and } \\
100 \% \text { after PHE. . }\end{array}$ \\
\hline $\begin{array}{l}\text { Glass et al. } \\
\text { [37] }\end{array}$ & $\begin{array}{l}N=543 \text { term } \\
\text { and preterm }\end{array}$ & $\begin{array}{l}N=284 \mathrm{HIE} \\
N=142 \\
\text { stroke } \\
N=108 \mathrm{IVH}\end{array}$ & $\begin{array}{l}N=508 \\
\text { PHB } \\
N=21 \text { LEV } \\
N=5 \text { PHE } \\
\text { (doses: } \mathrm{N} / \\
\text { A) }\end{array}$ & N/A & $\begin{array}{l}\text { 354/543 (66\%) neonates had incomplete response to the initial loading dose of } \\
\text { AED. } \\
\text { Incomplete response was similar for PHB (66\%), LEV (67\%), PHE (80\%). }\end{array}$ \\
\hline
\end{tabular}

$N$ number of patients; $P H B$ phenobarbital; PHE phenytoin; CNS central nervous system; $A E D$ anti-epileptic drug; N/A not available; HIE hypoxic ischemic encephalopathy; IVH intra-ventricular hemorrhage; BFNE benign familial neonatal epilepsy; IUGR intra-uterine growth restriction; MDZ midazolam; LID lidocaine; $B M T$ bumetanide; MAS meconium aspiration syndrome; RDS respiratory distress syndrome; LEV levetiracetam. EEG electroencephalography; $L R Z$ lorazepam; DZP diazepam; CFM cerebral function monitoring; NAS neonatal abstinence syndrome; APD acute placental detachment 
decision was made because most studies analyzed small populations, which were too small for statistical analysis; however, this method provided us with the opportunity to analyze the whole dataset as 1 large cohort of patients. Overall, 556 patients whit HIE, 45 patients whit stroke and 76 patients whit genetic channelopathies were considered.

\section{Limitations}

The primary limitation when interpreting study results was that most studies analyzed heterogeneous populations, including both full-term and preterm newborns, with seizures caused by a variety of etiologies, which prevented the assessment of relationships between seizure etiology and treatment efficacy, in most cases. In addition, no consensus regarding the definition of treatment efficacy is available in the literature; therefore, each study relied on a unique definition of efficacy, which ranged from a "seizure reduction of more than $80 \%$ within an hour from the drug administration," to "a global seizure burden reduction during the response period on EEG-monitoring", to "greater than $30 \%$ seizure reduction compared to another medication". The authors more often referred to an overall efficacy, without specifying the time from drug administration to seizure burden reduction as assessed by EEG monitoring.

Another limitation was that most of the available studies described combination treatments, resulting in the possible misinterpretation of each drug's specific effectiveness. In addition, as previously mentioned, we were unable to perform a meta-analysis because many studies included examined small, heterogeneous patient populations.

\section{Hypoxic-ischemic encephalopathy}

Among the studies reported in our review, 11 described homogeneous populations of newborns with HIE. Overall, 556 newborns with HIE were described (Table 5).

In a cohort of 76 asphyxiated newborns treated with phenobarbital, as a first-line anticonvulsant, efficacy was reported in $65 \%$ of cases, which is in line with previously available reports in the literature [33, 47, 48, 50, 87].

Among benzodiazepines, lorazepam was used as a first- or second-line anticonvulsant in a small cohort of 10 asphyxiated patients, with an overall $40 \%$ response; however, the 52 patients who did respond, were being treated simultaneously with phenobarbital, making it impossible to determine whether either drug individually or the combination was actually effective.

When used as a second-line anticonvulsant in 226 newborns after phenobarbital failure, midazolam was reported to be effective in $32 \%(14-50 \%)$ of the patients treated $[48,56]$. Reports from 107 newborns treated with Midazolam as a third-line treatment described an overall efficacy of 57.5\% [50]. These results appeared to be more promising during the first minutes after the initial administration (more than $80 \%$ seizure reduction within minutes after the first dose in 53 patients), but only half of the patients maintained seizure reduction over a 24-h period of observation. Serious hypotensive episodes were reported in $39(12 \%)$ patients treated.

A total of 317 patients were treated with lidocaine as a second- or third-line anticonvulsant, with a reported overall response to treatment of $45 \%$, when used as second-line treatment, $[52,56]$ and of $73.4 \%$, when used as a third-line anticonvulsant [52, 54]. Unfortunately, a closer look at the patient's features and hypoxia severity scores revealed that good responses were achieved in less severe cases, with no major structural brain damage. Patients with more critical injuries only displayed "partial responses" (less than 80\% seizure reduction) in 15\% of cases.

Bumetanide was used in an open-label clinical trial with promising results. Of the 14 patients treated, 5 had greater than $80 \%$ seizure reduction and 2 had greater than $50 \%$ seizure reduction after the first dose.22 When combined with phenobarbital, bumetanide resulted in significative seizure reduction in 5 additional patients. Unfortunately, although promising, the trial was stopped early due to ototoxicity concerns; ongoing trials are currently evaluating different treatment protocols and dosing regimens.

A cohort of 76 patients was treated with levetiracetam. As a first line anticonvulsant, used in 22 patients, levetiracetam was effective in providing seizure freedom to $50 \%$ of patients after $40 \mathrm{~h}$ and to $100 \%$ of patients between 100 and $120 \mathrm{~h}$ after the initiation of treatment. An overall $92 \%$ response rate to levetiracetam as a secondline anticonvulsant after phenobarbital failure was reported in the remaining 54 patients across the 2 studies $[46,47]$. When compared with the results obtained in the group treated with phenobarbital first, initial treatment with levetiracetam predicted a shorter interval to seizure freedom in both univariate and multivariate analyses, after adjusting for seizure frequency and HIE severity scores.

\section{Stroke}

Several patients with stroke were reported in different studies. Unfortunately, only data from 45 patients were evaluable for analysis because other patients belonged to larger cohorts that did not stratify results according to etiology (Table 6) $[52,55,65]$. These 45 patients were treated first with phenobarbital, but $43 / 45$ (95.5\%) patients required other anticonvulsants, suggesting that phenobarbital may be ineffective for treating strokerelated seizures. In non-responsive patients, 41 were treated with midazolam and lidocaine, as second- or 
Table 3 Full description of the sources: retrospective studies

\begin{tabular}{|c|c|c|c|c|c|}
\hline $\begin{array}{l}\text { Retrospective } \\
\text { studies }\end{array}$ & Population & Etiology & Treatment & Add-on therapy & Outcome \\
\hline $\begin{array}{l}\text { Abend et al. } \\
\text { [38] }\end{array}$ & $\begin{array}{l}N=23 \\
\text { late } \\
\text { preterm } \\
\text { and term }\end{array}$ & $\begin{array}{l}N=8 \mathrm{HIE} \\
N=4 \text { genetic } \\
N=3 \\
\text { malformative } \\
N=3 \\
\text { infections } \\
N=2 \\
C r y p t o g e n i c \\
N=2 \text { stroke } \\
N=1 \text { tumor }\end{array}$ & $\begin{array}{l}N=18 \text { PHB } \\
\text { (dose: } \mathrm{N} / \mathrm{A}) \\
N=1 \mathrm{PHE} \\
\text { (dose: N/A) } \\
N=4 \mathrm{LEV} \\
(10-20 \mathrm{mg} / \mathrm{kg})\end{array}$ & $\begin{array}{l}N=13 \text { LEV as } 2 \text { nd line } \\
\text { (dose: N/A) } \\
N=5 \text { LEV as 3rd line }\end{array}$ & $\begin{array}{l}\text { Seizure cessation in } 7 / 23(30 \%) \text {; seizure } \\
\text { reduction }(>50 \%) \text { in } 1 / 23 \text {. }\end{array}$ \\
\hline $\begin{array}{l}\text { Khan et al. } \\
\text { [39] }\end{array}$ & $\begin{array}{l}N=22 \\
\text { term }\end{array}$ & $\begin{array}{l}N=12 \mathrm{HIE} \\
N=2 \mathrm{IVH} \\
N=2 \mathrm{CNS} \\
\text { infections } \\
N=6 \text { various }\end{array}$ & $\begin{array}{l}N=16 \text { PHB } \\
\text { (dose: N/A) } \\
N=3 \text { LEV } \\
\text { (dose: N/A) }\end{array}$ & $\begin{array}{l}N=19 \text { received } L E V \text { as } 2 n d \\
(N=16), 3 r d(N=2) \text { or } 4 \text { th } \\
(N=1) \text { line }\end{array}$ & $\begin{array}{l}7 \text { of } 22 \text { patients (32\%) achieved complete } \\
\text { cessation } \\
\text { of seizures after administration of the } \\
\text { loading dose, } 14(64 \%) \text { achieved cessation } \\
\text { of seizures by } 24 \mathrm{~h}, 19(86 \%) \text { by } 48 \mathrm{~h} \text {, and all } \\
22(100 \%) \text { by } 72 \mathrm{~h}\end{array}$ \\
\hline $\begin{array}{l}\text { Khan et al. } \\
{[40]}\end{array}$ & $\begin{array}{l}N=12 \\
\text { preterm }\end{array}$ & $\begin{array}{l}N=5 \mathrm{HIE} \\
N=3 \mathrm{IVH} \\
N=3 \mathrm{~N} / \mathrm{A} \\
N=1 \mathrm{HSV} \\
\text { encephalitis }\end{array}$ & $\begin{array}{l}N=9 \text { PHB } \\
\text { (dose: N/A) } \\
N=3 \text { LID } \\
\text { (dose: N/A) }\end{array}$ & $\begin{array}{l}N=12 \text { LEV } \\
\text { (dose: N/A) }\end{array}$ & $\begin{array}{l}4 \text { patients ( } 36 \% \text { ) reached seizure cessation } \\
\text { after the loading dose, } 9 \text { ( } 82 \% \text { ) by } 24 \text { h, } 10 \\
\text { ( } 91 \%) \text { by } 48 \text { h, and } 10 \text { subjects ( } 91 \%) \text { by } 72 \\
\text { h. }\end{array}$ \\
\hline $\begin{array}{l}\text { Rakshabhuva- } \\
\text { nkar et al. } \\
\text { [41] }\end{array}$ & $\begin{array}{l}N=8 \\
\text { term and } \\
\text { preterm }\end{array}$ & $\begin{array}{l}N=5 \mathrm{HIE} \\
N=2 \mathrm{IVH} \\
N=1 \mathrm{~N} / \mathrm{A}\end{array}$ & $\begin{array}{l}N=8 \text { PHB } \\
\text { (dose: N/A) } \\
N=\text { N/A PHE } \\
\text { (dose: N/A) }\end{array}$ & $\begin{array}{l}N=8 \mathrm{LEV} \\
(10 \mathrm{mg} / \mathrm{kg})\end{array}$ & LEV's effectiveness in $6 / 8$ patients. \\
\hline $\begin{array}{l}\text { Lo Yee Yau } \\
\text { et al. } \\
{[42]}\end{array}$ & $\begin{array}{l}N=12 \\
\text { preterm } \\
\text { and term }\end{array}$ & $\begin{array}{l}N=6 \mathrm{HIE} \\
N=3 \mathrm{CNS} \\
\text { infections } \\
N=1 \\
\text { hypoglicemia } \\
N=2 \\
\text { metabolic }\end{array}$ & $\begin{array}{l}N=12 \mathrm{PHB} \\
\text { (dose: N/A) }\end{array}$ & $\begin{array}{l}\mathrm{N}=8 \mathrm{MDZ} \text { as } 2 \mathrm{nd} \text { line } \mathrm{AED} \\
\text { and LEV as 3rd line. } \\
\mathrm{N}=4 \text { were given LEV as } \\
\text { 2nd line AED. }\end{array}$ & $\begin{array}{l}\text { LEV's efficacy: } 75 \% \text { of patients treated. } \\
\text { No side effects reported. }\end{array}$ \\
\hline $\begin{array}{l}\text { Maljevic et al. } \\
{[43]}\end{array}$ & $N=10$ & $\begin{array}{l}\text { KCNQ } 3 \\
\text { mutations }\end{array}$ & $\begin{array}{l}N=1 \text { PYR } \\
N=2 \text { LEV } \\
N=1 \text { OXC }\end{array}$ & $\begin{array}{l}\mathrm{N}=1 \mathrm{LEV} \\
(65 \mathrm{mg} / \mathrm{kg}) \\
N=1 \mathrm{OXC} \\
(20 \mathrm{mg} / \mathrm{kg})\end{array}$ & $\begin{array}{l}1 \text { was seizure free after one dose of LEV. } \\
1 \text { was seizure free after day } 20 \text { on LEV. } \\
1 \text { was seizure free on OXC. }\end{array}$ \\
\hline $\begin{array}{l}\text { Shin et al. } \\
{[44]}\end{array}$ & $\begin{array}{l}N=18 \\
\text { term and } \\
\text { preterm }\end{array}$ & $\begin{array}{l}N=12 \mathrm{HIE} \\
N=1 \mathrm{CNS} \\
N=1 \mathrm{IVH} \\
N=4 \\
\text { malformative }\end{array}$ & $\begin{array}{l}N=18 \mathrm{PHB} \text { or } \mathrm{PHE} \\
\text { (dose: } \mathrm{N} / \mathrm{A} \text { ) }\end{array}$ & $\begin{array}{l}N=18 \text { LEV after PHB failure } \\
(N=1 \text { only LEV } \\
N=11 \text { LEV }+ \text { PHB } \\
N=6 \text { PHE }+ \text { LEV })\end{array}$ & $\begin{array}{l}94 \% \text { of patients had seizure cessation within } \\
\text { the first week from LEV's introduction, and } \\
89 \% \text { remained seizure-free under LEV } \\
\text { monotherapy at } 1 \text { month. }\end{array}$ \\
\hline $\begin{array}{l}\text { Han et al. } \\
{[45]}\end{array}$ & $\begin{array}{l}N=37 \\
\text { preterm }\end{array}$ & $\begin{array}{l}N=15 \mathrm{HIE} \\
N=6 \mathrm{GMH} \\
N=14 \mathrm{IVH} \\
N=1 \\
\text { malformative } \\
N=1 \\
\text { meningitis }\end{array}$ & $\begin{array}{l}\text { LEV } \\
(40-60 \mathrm{mg} / \mathrm{kg})\end{array}$ & $\begin{array}{l}\mathrm{N}=\mathrm{N} / \mathrm{A} \text { PHB } \\
(20 \mathrm{mg} / \mathrm{kg}) \\
\mathrm{N}=\mathrm{N} / \mathrm{A} \text { PHE, MDZ, TPM, VPA } \\
\text { as 3rd line. }\end{array}$ & $\begin{array}{l}\text { Seizure cessation in } 21 \text { patients (57\%) with } \\
\text { LEV alone. } \\
\text { Seizure cessation in } 9 \text { infants (24\%) after LEV } \\
+ \text { PHB. } \\
7 \text { required third-line AED. }\end{array}$ \\
\hline $\begin{array}{l}\text { Venkatesan } \\
\text { et al. } \\
{[46]}\end{array}$ & $\begin{array}{l}N=32 \\
\text { term }\end{array}$ & HIE & $\begin{array}{l}N=23 \text { PHB } \\
N=2 \text { LEV } \\
N=2 \text { MDZ } \\
\text { (doses: N/A) }\end{array}$ & $\begin{array}{l}N=23 \text { LEV as } 2 \text { nd line } \\
N=2 \text { LEV as } 3 \text { rd line after } \\
\text { PHB and MDZ failure }\end{array}$ & $\begin{array}{l}84 \% \text { of the patients treated with LEV } \\
\text { achieved seizure cessation within } 72 \mathrm{~h} \text {. }\end{array}$ \\
\hline $\begin{array}{l}\text { Rao et al. } \\
{[47]}\end{array}$ & $\begin{array}{l}N=44 \\
\text { term }\end{array}$ & HIE & $\begin{array}{l}N=23 \mathrm{PHB} \\
N=2 \mathrm{LEV}\end{array}$ & $N=10 \mathrm{LEV}$ as $2 \mathrm{nd}$ line & $\begin{array}{l}50 \% \text { of patients treated with levetiracetam } \\
\text { became seizure-free after } 40 \mathrm{~h} \text {, and } 100 \% \\
\text { achieved seizure freedom between } 100 \text { and } \\
120 \mathrm{~h} \text {. }\end{array}$ \\
\hline $\begin{array}{l}\text { Van der Broek } \\
\text { et al. } \\
{[48]}\end{array}$ & $\begin{array}{l}N=31 \\
\text { term }\end{array}$ & HIE & $\begin{array}{l}N=31 \mathrm{PHB} \\
(20 \mathrm{mg} / \mathrm{kg})\end{array}$ & / & PHB's efficacy: $66 \%$. \\
\hline $\begin{array}{l}\text { Boylan et al. } \\
\text { [49] }\end{array}$ & $\begin{array}{l}N=14 \\
\text { term and }\end{array}$ & $\begin{array}{l}N=4 \mathrm{HIE} \\
N=3 \mathrm{IVH}\end{array}$ & $\begin{array}{l}N=14 \mathrm{PHB} \\
(20-40 \mathrm{mg} / \mathrm{kg})\end{array}$ & $\begin{array}{l}N=4 \mathrm{CLZ} \\
N=\mathrm{N} / \mathrm{A} P H E\end{array}$ & $\begin{array}{l}\text { PHB was only effective in } 29 \% \text { of patients, } \\
\text { those with normal background EEGs or mild }\end{array}$ \\
\hline
\end{tabular}


Table 3 Full description of the sources: retrospective studies (Continued)

\begin{tabular}{|c|c|c|c|c|c|}
\hline Retrospective & Population & Etiology & Treatment & Add-on therapy & Outcome \\
\hline & preterm & $\begin{array}{l}N=3 \\
\text { metabolic } \\
N=1 \\
\text { meningitis } \\
N=3 \text { mild } \\
\text { asphyxia }\end{array}$ & & (doses: N/A) & $\begin{array}{l}\text { to moderate } \\
\text { background abnormalities and low seizure } \\
\text { burden. }\end{array}$ \\
\hline $\begin{array}{l}\text { Spagnoli } \\
\text { et al. } \\
{[50]}\end{array}$ & $\begin{array}{l}N=91 \\
\text { term and } \\
\text { preterm }\end{array}$ & $\begin{array}{l}N=45 \mathrm{HIE} \\
N=21 \mathrm{IVH} \\
N=4 \\
\text { malformative } \\
N=12 \\
\text { metabolic } \\
\text { disorders } \\
N=4 \mathrm{CNS} \\
\text { infection } \\
N=1 \text { genetic } \\
N=4 \mathrm{~N} / \mathrm{A}\end{array}$ & $\begin{array}{l}N=91 \mathrm{PHB} \\
(20 \mathrm{mg} / \mathrm{kg})\end{array}$ & $\begin{array}{l}\mathrm{N}=\mathrm{N} / \mathrm{A} \text { PHE } 20 \mathrm{mg} / \mathrm{kg} \text { as } \\
\text { 2nd line } \\
N=\mathrm{N} / \mathrm{A} \text { MDZ } 0.15 \mathrm{mg} / \mathrm{kg} \text { as } \\
\text { 3rd line }\end{array}$ & $\begin{array}{l}\text { PHB was effective alone in } 62.6 \% \text { of } \\
\text { patients. }\end{array}$ \\
\hline $\begin{array}{l}\text { Hakeem et al. } \\
{[51]}\end{array}$ & $\begin{array}{l}N=11 \\
\text { term and } \\
\text { preterm }\end{array}$ & $\begin{array}{l}N=6 \mathrm{HIE} \\
N=3 \mathrm{IVH} \\
N=1 \mathrm{CNS} \\
N=1 \mathrm{~N} / \mathrm{A}\end{array}$ & $\begin{array}{l}N=2 \text { oral cloral hydrate } \\
(30 \mathrm{mg} / \mathrm{kg}) \\
N=7 \mathrm{PHB} \\
\text { (20 mg/kg) } \\
N=2 \mathrm{DZP} \\
\text { (1 to } 2 \mathrm{mg} \text { iv bolus) }\end{array}$ & $\begin{array}{l}N=1 \mathrm{CLZ} \text { c.i. }(10 \mathrm{mcg} / \mathrm{kg} / \\
\mathrm{hr})\end{array}$ & $\begin{array}{l}6 / 7 \text { responded to PHB but } 4 / 7 \text { later } \\
\text { relapsed. }\end{array}$ \\
\hline $\begin{array}{l}\text { Weeke et al. } \\
\text { [52] }\end{array}$ & $\begin{array}{l}N=413 \\
(N=319 \\
\text { term, } N= \\
94 \text { preterm })\end{array}$ & $\begin{array}{l}N=228 \mathrm{HIE} \\
N=45 \mathrm{HIE} \\
N=32 \text { PAIS } \\
N=40 \text { CNS } \\
\text { infections } \\
N=100 \\
\text { others }\end{array}$ & $\begin{array}{l}N=413 \mathrm{PHB} \\
\text { (dose: } 20 \mathrm{mg} / \mathrm{kg} \text { ) }\end{array}$ & $\begin{array}{l}N=186 \text { LID as } 2 \text { nd line } \\
N=172 \text { LID as 3rd line }\end{array}$ & $\begin{array}{l}\text { In term infants, a response to LID was seen } \\
\text { in } 72.5-80 \% \text {, with cessation of seizures and } \\
\text { no need for rescue AED in } 21.4-67.6 \% \text {. } \\
\text { Lower response rate in preterm (55.5-58.2\% } \\
\text { with cessation of seizures and no other AED } \\
\text { in only } 16.4-40.7 \% \text { ). }\end{array}$ \\
\hline $\begin{array}{l}\text { Lundqvist } \\
\text { et al. } \\
\text { [53] }\end{array}$ & $\begin{array}{l}N=30 \\
\text { term }\end{array}$ & $\begin{array}{l}N=18 \mathrm{HIE} \\
N=4 \\
\text { meningitis } \\
N=6 \text { PAIS } \\
N=1 \\
\text { hypoglicemia } \\
N=1 \\
\text { uncertain }\end{array}$ & $\begin{array}{l}N=17 \mathrm{DZP} \\
\text { (dose: N/A) } \\
N=8 \mathrm{MDZ} \\
\text { (dose: N/A) } \\
N=5 \mathrm{DZP}+\mathrm{MDZ} \\
\text { (dose: N/A) }\end{array}$ & $N=30 \mathrm{LID}$ & LID's efficacy: $65 \%$. \\
\hline $\begin{array}{l}\text { Van der Broek } \\
\text { et al. } \\
{[54]}\end{array}$ & $\begin{array}{l}N=22 \\
\text { term }\end{array}$ & HIE & $\begin{array}{l}N=22 \mathrm{PHB} \\
\text { (dose: N/A) }\end{array}$ & $\begin{array}{l}N=22 \mathrm{MDZ} \\
\text { (dose: N/A) } \\
N=22 \mathrm{LID} \\
\text { (dose: } 2 \mathrm{mg} / \mathrm{kg} \text {, followed } \\
\text { by } 4 \mathrm{mg} / \mathrm{kg} / \mathrm{h} \text { c.i.) }\end{array}$ & $\begin{array}{l}20 / 22(90 \%) \text { newborns responded to LID. } \\
\text { No cardiac arrythmias were reported } \\
(91 \%)\end{array}$ \\
\hline $\begin{array}{l}\text { Jennekens } \\
\text { et al. } \\
{[55]}\end{array}$ & $\begin{array}{l}N=11 \\
\text { term }\end{array}$ & $N=11$ stroke & $\begin{array}{l}N=11 \mathrm{PHB} \\
(20 \mathrm{mg} / \mathrm{kg})\end{array}$ & $\begin{array}{l}N=8 \mathrm{MDZ} \text { as } 2 \mathrm{nd} \text { line } \\
N=9 \mathrm{LID} \text { as } 3 \mathrm{rd} \text { line }\end{array}$ & $\begin{array}{l}\text { In term newborns with PAIS, MDZ and LID } \\
\text { induce a shift from lower to higher } \\
\text { frequency electrocortical activity. Compared } \\
\text { to LID, MDZ reduced more pronouncedly } \\
\text { the total EEG power. }\end{array}$ \\
\hline $\begin{array}{l}\text { Shany et al. } \\
{[56]}\end{array}$ & $\begin{array}{l}N=30 \\
\text { term }\end{array}$ & $N=30 \mathrm{HIE}$ & $N=30 \mathrm{DZP}$ or $\mathrm{PHB}$ & $\begin{array}{l}N=22 \mathrm{LID} \text { as } 2 \mathrm{nd} \text { line } \\
N=8 \mathrm{MDZ} \text { as } 2 \text { nd line }\end{array}$ & $77 \%$ response rate to LID. \\
\hline $\begin{array}{l}\text { McDermott } \\
\text { et al. } \\
{[57]}\end{array}$ & $\begin{array}{l}N=10 \\
\text { term }\end{array}$ & HIE & $\begin{array}{l}N=5 \mathrm{LRZ} \\
N=4 \text { PHB or PHE }(20 \mathrm{mg} / \\
\mathrm{kg}) \\
N=1 \mathrm{PHB} \\
(20 \mathrm{mg} / \mathrm{kg})\end{array}$ & $N=5 L R Z$ as 2 nd line & $\begin{array}{l}\text { Administration of a single dose of } \\
\mathrm{LRZ} \text { stopped seizures in all neonates. } 4 \\
\text { neonates receiving simultaneously PHB and/ } \\
\text { or PHE } \\
\text { had no further seizures. } 6 \text { had seizure } \\
\text { recurrence. }\end{array}$ \\
\hline $\begin{array}{l}\text { Castro Conde } \\
\text { et al. } \\
{[58]}\end{array}$ & $\begin{array}{l}N=13 \\
\text { term and } \\
\text { preterm }\end{array}$ & $\begin{array}{l}N=7 \mathrm{HIEN}= \\
3 \text { stroke } \\
N=2 \mathrm{IVH} \\
N=1 \mathrm{~N} / \mathrm{A}\end{array}$ & $\begin{array}{l}\mathrm{N}=32 \mathrm{PHB}(20 \mathrm{mg} / \mathrm{kg} \\
\text { tritated up to } 40 \mathrm{mg} / \mathrm{kg}) \\
\text { followed by PHE as } 2 \mathrm{nd} \\
\text { line AED }(20 \mathrm{mg} / \mathrm{kg})\end{array}$ & $\begin{array}{l}N=13 \mathrm{MDZ} \text { as } 2 \mathrm{nd}(9 / 13) \\
\text { or 3rd }(4 / 13) \text { line } \mathrm{AED} \text { in } \\
\text { non-responders. }\end{array}$ & $\begin{array}{l}\text { Ten of } 13 \text { neonates with SE treated with } \\
\text { midazolam } \\
\text { were electrically controlled in the first hour } \\
\text { of treatment. }\end{array}$ \\
\hline $\begin{array}{l}\text { Vilan et al. } \\
{[59]}\end{array}$ & $\begin{array}{l}N=9 \\
\text { term }\end{array}$ & $\begin{array}{l}\text { KCNQ2 } \\
\text { mutations }\end{array}$ & $N=9 \mathrm{PHB}$ & $\begin{array}{l}N=8 \mathrm{PYR}, N=6 \mathrm{LID} \\
N=6 \mathrm{MDZ}, N=3 \mathrm{CZP}, N=3\end{array}$ & $\begin{array}{l}\text { PH and PYR (used in } 8 / 9 \text { patients) were } \\
\text { ineffective. }\end{array}$ \\
\hline
\end{tabular}


Table 3 Full description of the sources: retrospective studies (Continued)

\begin{tabular}{|c|c|c|c|c|c|}
\hline $\begin{array}{l}\text { Retrospective } \\
\text { studies }\end{array}$ & Population & Etiology & Treatment & Add-on therapy & Outcome \\
\hline & & & & $\begin{array}{l}\text { LEV, } \\
N=2 \text { PHE, } N=2 \text { VPA } N=2 \\
C B Z, N=1 \text { TPM }\end{array}$ & $\begin{array}{l}2 \text { patients were SF during LID infusion and } \\
\text { were later switched to oral PHT or oral CBZ. }\end{array}$ \\
\hline $\begin{array}{l}\text { Montesclaros } \\
\text { Hortigüela } \\
\text { et al. } \\
{[60]}\end{array}$ & $\begin{array}{l}N=13 \\
\text { G.A. }=N / A\end{array}$ & $\begin{array}{l}\text { KCNQ2 } \\
\text { mutations }\end{array}$ & $\begin{array}{l}N=10 \text { PHB } \\
N=2 \text { LEV } \\
N=1 \mathrm{MDZ} \\
\text { (doses: N/A) }\end{array}$ & $\begin{array}{l}\text { Several AED were } \\
\text { administered as 2nd line: } \\
\text { CBZ, LEV, MDZ, VPA, PHE, } \\
\text { TPM, VGB, LID, OXC, PYR }\end{array}$ & $\begin{array}{l}5 / 9 \text { were seizure free but with severe } \\
\text { impairment in psychomotor development } \\
\text { in treatment with CBZ }(n=2), \mathrm{VPA}+\mathrm{CBZ}+ \\
\mathrm{LCM}(n=1), \mathrm{PHB}+\mathrm{VPA}+\mathrm{OXC}(n=1) \\
\text { OXC + TPM }(n=1) \text {. }\end{array}$ \\
\hline $\begin{array}{l}\text { Pisano et al. } \\
\text { [61] }\end{array}$ & $\begin{array}{l}N=15 \\
\text { term }\end{array}$ & $\begin{array}{l}\text { KCNQ2 } \\
\text { mutations }\end{array}$ & $\begin{array}{l}\text { Multiple AEDs (including } \\
\text { PHB as first-line AED, VPA, } \\
\text { steroids) were tried } \\
\text { unsuccessfully }\end{array}$ & $\begin{array}{l}N=\mathrm{N} / \mathrm{A} C \mathrm{CBZ} \\
(20 \mathrm{mg} / \mathrm{kg} / \mathrm{day}) \\
N=\mathrm{N} / \mathrm{A} \mathrm{PHE} \\
\text { (dose widely ranging) }\end{array}$ & $\begin{array}{l}53 \% \text { of the patients were seizure-free on } \\
\text { CBZ; } \\
33 \% \text { responded to PHE; } \\
\text { the remaining } 47 \% \text { of the patients } \\
\text { responded to TPM and LEV. }\end{array}$ \\
\hline $\begin{array}{l}\text { Sands et al. } \\
\text { [62] }\end{array}$ & $\begin{array}{l}N=19 \\
\text { term }\end{array}$ & $\begin{array}{l}\text { SLC13A5 } \\
\text { mutations } \\
\text { (KCNQ2 } \\
\text { gene) }\end{array}$ & $\begin{array}{l}N=13 \mathrm{PHB} \\
\text { (dose: N/A) } \\
N=4 \mathrm{CBZ} \\
(10 \mathrm{mg} / \mathrm{kg})\end{array}$ & $\begin{array}{l}N=15 \mathrm{CBZ} \\
(10 \mathrm{mg} / \mathrm{kg})\end{array}$ & CBZ's efficacy: 89\%. \\
\hline $\begin{array}{l}\text { Singh et al. } \\
{[63]}\end{array}$ & $\begin{array}{l}N=10 \\
\text { term }\end{array}$ & $\begin{array}{l}N=8 \mathrm{HIE} \\
N=2 \\
\text { unknown }\end{array}$ & $\begin{array}{l}N=10 C B Z \\
\text { (dose: } 10 \mathrm{mg} / \mathrm{kg} \text { ) }\end{array}$ & $N=2 \mathrm{DZP}$ & $\begin{array}{l}\text { Seizure control in } 80 \% \text { of patients on CBZ; } \\
2 \text { patients needed DZP as } 2 \text { nd line. }\end{array}$ \\
\hline $\begin{array}{l}\text { Glass et al. } \\
\text { [64] }\end{array}$ & $\begin{array}{l}\mathrm{N}=6 \\
\text { term }\end{array}$ & $\mathrm{HIE}$ & $\begin{array}{l}\mathrm{N}=5 \mathrm{PHB} \\
(30-60 \mathrm{mg} / \mathrm{kg})\end{array}$ & $\begin{array}{l}\mathrm{N}=5 \mathrm{TPM} \\
(10 \mathrm{mg} / \mathrm{kg})\end{array}$ & $\begin{array}{l}3 / 5 \text { patients treated with TPM had seizure } \\
\text { reduction or cessation. One adjunctive } \\
\text { patient achieved seizure freedom on TPM at } \\
6 \text { months. }\end{array}$ \\
\hline
\end{tabular}

$N$ number of patients; $P H B$ phenobarbital; PHE phenytoin; CNS central nervous system; $A E D$ anti-epileptic drug; N/A not available; HIE hypoxic ischemic encephalopathy; IVH intra-ventricular hemorrhage; BFNE benign familial neonatal epilepsy; IUGR intra-uterine growth restriction; MDZ midazolam; LID lidocaine; $B M T$ bumetanide; MAS meconium aspiration syndrome; $R D S$ respiratory distress syndrome; $L E V$ levetiracetam. $E E G$ electroencephalography; $L R Z$ lorazepam; $D Z P$ diazepam; CFM cerebral function monitoring; NAS neonatal abstinence syndrome; VPA valproic acid; CBZ carbamazepine; TPM topiramate; G.A. gestational age; SE status epilepticus; HSV herpes simplex virus; PYR pyridoxine; OXC oxcarbazepine; GMH germinal matrix hemorrhage; TPM topiramate; CLZ clonazepam; DZP diazepam; LRZ lorazepam; PAIS perinatal arterial ischemic stroke; SF seizure- free

third-line anticonvulsants. In 9 of these patients, the spectral aEEG properties were analyzed, and no data on the clinical efficacy of the administered drugs were available. However, the authors reported that midazolam administration resulted in the moderate suppression of background EEG activity within minutes after the first administration, which lasted for 30-60 min. In contrast, lidocaine administration resulted in a more moderate suppression of background activity and has been reported to suppress electrical activity more strongly within ischemic areas of the brain, suggesting that lidocaine may be more specific and effective for the treatment of specific seizure related etiology [55]. Similar results were reported for the remaining 32 patients with stroke, with lidocaine administration resulting in seizure control in $27 / 32$ patients (84\%), compared with the less promising efficacy of midazolam, which was only effective in $5 / 32(16 \%)$ of the patients treated [52]. Interestingly, the authors reported that the efficacy of lidocaine appeared to be higher in full-term newborns than in preterm newborns and that efficacy appeared to be higher when used as a second-line anticonvulsant after phenytoin, rather than as a third-line AED. One patient with a stroke was reported to have been treated with levetiracetam as a third-line anticonvulsant after phenobarbital and phenytoin failure, with electric remission of seizures $17 \mathrm{~min}$ after drug administration [65].

No side effects have been reported associated with the administration of lidocaine or midazolam for stroke patients; however, no long-term follow-up was provided except for the single patient treated with levetiracetam, who was reported to be successfully maintaining seizure-freedom on levetiracetam monotherapy at an 18month follow-up.

\section{Genetic Channelopathies}

Mutations in genes that encode neuronal ion channels have been associated with a number of early-onset epileptic encephalopathies. A total of 76 patients among case reports, retrospective, and prospective studies were collected in our review (Table 7). In line with the literature, KCNQ2 mutations represented the most common genetic anomalies, associated with early-onset seizures in $86 \%$ of the patients included in this review, followed by KCNQ3 mutations (8\%) [43, 59-62, 71-76, 85, 86].

Among these 76 patients, 74 were treated with sodium channel blockers, including carbamazepine, phenytoin, lidocaine, and oxcarbazepine, during the courses of their 
Table 4 Full description of the sources: case reports

\begin{tabular}{|c|c|c|c|c|c|}
\hline & Population & Etiology & Treatment & Add-on therapy & Outcome \\
\hline $\begin{array}{l}\text { Shoemaker } \\
\text { et al. } \\
{[65]}\end{array}$ & $\begin{array}{l}N=3 \\
\text { term and } \\
\text { preterm }\end{array}$ & $\begin{array}{l}N=1 \text { PAIS } \\
N=2 \text { PHVD }\end{array}$ & $\begin{array}{l}N=2 \text { PHB and } \\
\text { PHE } \\
\text { (dose: N/A) } \\
N=1 \text { PHE and } \\
\text { OXC } \\
\text { (dose: N/A) }\end{array}$ & $\begin{array}{l}N=3 \text { LEV as 3rd line } \\
\text { (dose: N/A) }\end{array}$ & $\begin{array}{l}\text { LEV's administration resulted in seizure control in } \\
\text { all three patients. }\end{array}$ \\
\hline $\begin{array}{l}\text { Tanriverdi } \\
\text { et al. } \\
\text { [66] }\end{array}$ & $\begin{array}{l}N=1 \\
\text { term }\end{array}$ & SWS & $\begin{array}{l}\text { PHB } \\
(20 \mathrm{mg} / \mathrm{kg})\end{array}$ & $\begin{array}{l}\text { PHE as } 2 \mathrm{nd} \text { line } \\
(20 \mathrm{mg} / \mathrm{kg}) \\
\text { LEV as } 3 \mathrm{rd} \text { line } \\
(20 \mathrm{mg} / \mathrm{kg})\end{array}$ & $\begin{array}{l}\text { Seizure control was achieved after LEV } \\
\text { intravenous infusion. }\end{array}$ \\
\hline $\begin{array}{l}\text { Hmaimess } \\
\text { et al. } \\
{[67]}\end{array}$ & $\begin{array}{l}N=1 \\
\text { Term }\end{array}$ & KCNT1 mutation & $\begin{array}{l}\text { PHB } \\
\text { (dose: N/A) }\end{array}$ & $\begin{array}{l}\text { PHT, LTG, CLZ } \\
\text { LEV } \\
(10-30 \mathrm{mg} / \mathrm{kg})\end{array}$ & $\begin{array}{l}\text { LEV's introduction resulted in dramatic } \\
\text { decrease in seizure activity by the eighth day of } \\
\text { treatment. }\end{array}$ \\
\hline $\begin{array}{l}\text { Ledet et al. } \\
\text { [68] }\end{array}$ & $\begin{array}{l}N=1 \\
\text { term }\end{array}$ & LLA & $\begin{array}{l}\text { PHB } \\
(20 \mathrm{mg} / \mathrm{kg})\end{array}$ & $\begin{array}{l}\text { LEV } \\
(40 \mathrm{mg} / \mathrm{kg})\end{array}$ & $\begin{array}{l}\text { The patient was seizure-free on PHB and main- } \\
\text { tained seizure freedom on LEV that minimally in- } \\
\text { terfered with her other ongoing treatments. }\end{array}$ \\
\hline $\begin{array}{l}\text { Li Jiang } \\
\text { et al. } \\
{[69]}\end{array}$ & $\begin{array}{l}N=9 \\
\text { term }\end{array}$ & STXBP1mutations & $\begin{array}{l}\text { PHB } \\
\text { (dose: N/A) }\end{array}$ & $\begin{array}{l}5 \text { patients did not respond and } \\
\text { were tried on several AEDs (TPM, } \\
\text { NZP, LEV, VPA, VIT B6, PDN, ACTH, } \\
\text { KD) }\end{array}$ & $\begin{array}{l}44.4 \% \text { of cases }(4 / 9) \text { in our study showed } \\
\text { apparent responses to LEV. }\end{array}$ \\
\hline $\begin{array}{l}\text { Dilena } \\
\text { et al. } \\
{[70]}\end{array}$ & $\begin{array}{l}N=1 \\
\text { term }\end{array}$ & SCN2A & $\begin{array}{l}\text { PHB } \\
\text { (dose: N/A) }\end{array}$ & $\begin{array}{l}\text { LEV + PYR as } 2 \text { nd line } \\
\text { PHE as } 3 \mathrm{rd} \text { line } \\
\text { (12-18 } \mathrm{mg} / \mathrm{kg} / \text { day })\end{array}$ & $\begin{array}{l}\text { Seizure freedom was reached on PHE, first, and } \\
\text { maintained on oral CBZ. }\end{array}$ \\
\hline $\begin{array}{l}\text { Bonhorst } \\
\text { et al. } \\
\text { [71] }\end{array}$ & $\begin{array}{l}N=1 \\
\text { term }\end{array}$ & KCNQ2 & $\begin{array}{l}\text { PHB } \\
(20 \mathrm{mg} / \mathrm{kg}) \\
+ \\
\text { VIT B6 } \\
(30 \mathrm{mg} / \mathrm{kg} / \mathrm{d})\end{array}$ & $\begin{array}{l}\text { MDZ c.i. as } 2 \text { nd line } \\
\text { ( } 0.25 \mathrm{mg} / \mathrm{kg} / \mathrm{h}) \\
\text { TPM as } 3 \mathrm{rd} \text { line } \\
\text { ( } 2 \mathrm{mg} / \mathrm{kg} / \mathrm{day}) \\
\text { LID }(6 \mathrm{mg} / \mathrm{kg}) \text {, then switched to } \\
\text { PHE and, later CBZ (dose: N/A) }\end{array}$ & $\begin{array}{l}\text { Seizure freedom was reached on LID; the patient } \\
\text { developed methemoglobinemia as side-effect } \\
\text { and seizure freedom was maintained with PHE, } \\
\text { first, and oral CBZ, later. }\end{array}$ \\
\hline $\begin{array}{l}\text { Numis } \\
\text { et al. } \\
{[72]}\end{array}$ & $\begin{array}{l}N=3 \\
\text { term and } \\
\text { late } \\
\text { preterm }\end{array}$ & $\begin{array}{l}\text { KCNQ2 } \\
\text { encephalopathy }\end{array}$ & $\begin{array}{l}\text { PHB } \\
\text { (dose: N/A) }\end{array}$ & $\begin{array}{l}\text { LEV, TPM, VGB, CLZ, KD failed. } \\
\text { CBZ was initiated at } 3,4 \text { and } 13 \\
\text { months } \\
\text { (dose: N/A) }\end{array}$ & $\begin{array}{l}\text { 2/3 patients responded to CBZ and were seizure } \\
\text { free at } 30 \text { months though developed severe } \\
\text { psychomotor delay, quadriplegia, axial hypotonia } \\
\text { with appendicular hypertonia, and a tendency to } \\
\text { opisthotonos. }\end{array}$ \\
\hline $\begin{array}{l}\text { Spagnoli } \\
\text { et al. } \\
{[73]}\end{array}$ & $\begin{array}{l}N=1 \\
\text { term }\end{array}$ & $\begin{array}{l}\text { EIMFS due to } \\
\text { KCNQ2 } \\
\text { mutations }\end{array}$ & $\begin{array}{l}\text { PHB } \\
\text { (dose N/A) }\end{array}$ & $\begin{array}{l}\text { PYR, LEV, PHE, MDZ, TPM, NTZ } \\
\text { CBZ } \\
\text { (dose: N/A) }\end{array}$ & $\begin{array}{l}\text { After multiple AEDs failure, seizure ceased after } \\
3 \text { weeks from CBZ's introduction. Patient was } \\
\text { seizure free at nine months. }\end{array}$ \\
\hline $\begin{array}{l}\text { Blumkin } \\
\text { et al. } \\
\text { [74] }\end{array}$ & $\begin{array}{l}N=1 \\
\text { Term }\end{array}$ & KNCQ2 & $\begin{array}{l}\text { PHB (dose: N/ } \\
\text { A) }\end{array}$ & $\begin{array}{l}\text { TPM, LEV, VPA, LTG, PYR, folinic } \\
\text { acid } \\
\text { CBZ } \\
(50 \mathrm{mg} / \mathrm{kg})\end{array}$ & $\begin{array}{l}\text { Seizure control was initially achieved with TPM. } \\
\text { Seizures reoccurred after } 3 \text { weeks and did not } \\
\text { respond to several AEDs until CBZ. }\end{array}$ \\
\hline $\begin{array}{l}\text { Buttle et al. } \\
{[75]}\end{array}$ & $\begin{array}{l}N=1 \\
\text { term }\end{array}$ & KCNQ2 & $\begin{array}{l}\text { PHB } \\
\text { (dose: N/A) }\end{array}$ & $\begin{array}{l}\text { LEV, LRZ, CLZ, PYR } \\
\text { LID } \\
(2-4 \mathrm{mg} / \mathrm{kg} / \mathrm{h}) \\
\text { CBZ } \\
(40 \mathrm{mg} / \mathrm{kg})\end{array}$ & $\begin{array}{l}\text { After several AEDs failed, seizure freedom was } \\
\text { reached on LID and maintained at a } 13 \text { months } \\
\text { follow-up on oral CBZ. }\end{array}$ \\
\hline $\begin{array}{l}\text { Soldovieri } \\
\text { et al. } \\
{[76]}\end{array}$ & $\begin{array}{l}N=1 \\
\text { term }\end{array}$ & $\begin{array}{l}\text { KCNQ2 mutation } \\
\text { (Kv7.2 subunit) }\end{array}$ & $\begin{array}{l}\text { PHB } \\
\text { (dose: N/A) }\end{array}$ & PYR, LEV, PHE, TPM, OXC & $\begin{array}{l}\text { Partial response to an association of PHB, PHE, } \\
\text { TPM. At } 5 \text { months he was switched to OXC and } \\
\text { maintained seizure freedom until } 14 \text { months. The } \\
\text { patient developed severe DD. }\end{array}$ \\
\hline $\begin{array}{l}\text { McNally } \\
\text { et al. } \\
\text { [77] }\end{array}$ & $\begin{array}{l}N=1 \\
\text { term }\end{array}$ & SCN8A & $\begin{array}{l}\text { PHB } \\
(20 \mathrm{mg} / \mathrm{kg}) \\
+ \\
\text { LEV } \\
(20-60 \mathrm{mg} / \mathrm{kg})\end{array}$ & $\begin{array}{l}\text { OXC } \\
\text { (up to } 80 \mathrm{mg} / \mathrm{kg} \text { ) } \\
+ \\
\text { PHE } \\
(20 \mathrm{mg} / \mathrm{kg}) \\
+ \\
\text { LTG } \\
(2 \mathrm{mg} / \mathrm{kg} / \text { day })\end{array}$ & $\begin{array}{l}\text { The association of three sodium channel } \\
\text { blockers (OXC + PHE + LTG) reduced seizures' } \\
\text { frequency. }\end{array}$ \\
\hline $\begin{array}{l}\text { Okumura } \\
\text { et al. } \\
\text { [78] }\end{array}$ & $\begin{array}{l}N=1 \\
\text { term }\end{array}$ & $\begin{array}{l}\text { 2q21- } \\
\text { q31deletion } \\
\text { (SCN1A cluster) }\end{array}$ & $\begin{array}{l}\text { PHB } \\
\text { (dose: } 20 \mathrm{mg} / \\
\mathrm{kg} \text { ) }\end{array}$ & $\begin{array}{l}\text { LEV as } 2 \mathrm{nd} \text { line } \\
(40 \mathrm{mg} / \mathrm{kg}) \\
\text { VPA as } 3 \mathrm{rd} \text { line }\end{array}$ & $\begin{array}{l}\text { PHB and LEV failed to control seizures; } \\
\text { VPA reduced seizures' frequency. }\end{array}$ \\
\hline
\end{tabular}


Table 4 Full description of the sources: case reports (Continued)

\begin{tabular}{|c|c|c|c|c|c|}
\hline & Population & Etiology & Treatment & Add-on therapy & Outcome \\
\hline & & & & $(50 \mathrm{mg} / \mathrm{kg})$ & \\
\hline $\begin{array}{l}\text { Riesgo } \\
\text { et al. } \\
{[79]}\end{array}$ & $\begin{array}{l}N=3 \\
\text { preterm } \\
\text { and term }\end{array}$ & $\begin{array}{l}N=1 \mathrm{NAS} \\
N=1 \text { fetal } \\
\text { distress } \\
N=1 \mathrm{PVL}\end{array}$ & $\begin{array}{l}\text { PHB } \\
\text { (dose: N/A) }\end{array}$ & $\begin{array}{l}\text { TPM } \\
(0.5-8 \mathrm{mg} / \mathrm{kg} / \mathrm{d}) \\
\text { after several other AEDs failed } \\
(\text { PHB, PHE, CLZ, VPA, MDZ) }\end{array}$ & $\begin{array}{l}\text { Seizure cessation in all three after TPM's } \\
\text { administration. }\end{array}$ \\
\hline $\begin{array}{l}\text { Sirsi et al. } \\
{[80]}\end{array}$ & $\begin{array}{l}N=3 \\
\text { term }\end{array}$ & $\begin{array}{l}N=1 \text { HIE } \\
N=1 \text { meningitis } \\
N=1 \text { EIEE }\end{array}$ & $\begin{array}{l}\text { PHB } \\
\text { (dose: N/A) }\end{array}$ & $\begin{array}{l}\text { PHE as } 2 \text { nd line } \\
\text { (dose: N/A) } \\
\text { MDZ as } 3 \text { rd line } \\
\text { (up to } 0.2 \mathrm{mcg} / \mathrm{kg} / \mathrm{h} \text { ) }\end{array}$ & $\begin{array}{l}\text { Seizure control within } 6-72 \mathrm{~h} \text { after MDZ's } \\
\text { introduction. } \\
\text { One patient developed hypotension, that } \\
\text { responded to inotropic support. }\end{array}$ \\
\hline $\begin{array}{l}\text { Steinberg } \\
\text { et al. } \\
{[81]}\end{array}$ & $\begin{array}{l}N=2 \\
\text { preterm }\end{array}$ & $\begin{array}{l}N=1 \mathrm{IVHN}=1 \\
\mathrm{PVL}\end{array}$ & $\begin{array}{l}\text { PHB } \\
(20 \mathrm{mg} / \mathrm{kg})\end{array}$ & $\begin{array}{l}\text { PHE as } 2 \mathrm{nd} \text { line } \\
(20 \mathrm{mg} / \mathrm{kg}) \\
\text { Rectal VPA as } 3 \mathrm{rd} \text { line } \\
(20-30 \mathrm{mg} / \mathrm{kg})\end{array}$ & $\begin{array}{l}\text { Seizure control was achieved and maintained on } \\
\text { a } 12 \text { months follow-up on VPA. }\end{array}$ \\
\hline $\begin{array}{l}\text { Tarocco } \\
\text { et al. } \\
{[82]}\end{array}$ & $\begin{array}{l}N=1 \\
\text { late } \\
\text { preterm }\end{array}$ & $\begin{array}{l}\text { Pierre-Robin, } \\
\text { polymicrogyria, } \\
\text { lissencephaly }\end{array}$ & $\begin{array}{l}\text { PHB } \\
\text { (dose: N/A) }\end{array}$ & $\begin{array}{l}\text { PHE, MDZ, LEV, PPF } \\
\text { KTM }(2 \mathrm{mg} / \mathrm{kg}+\text { c.i. of } 10 \mathrm{mcg} / \mathrm{kg} / \\
\text { min })\end{array}$ & $\begin{array}{l}\text { Immediate complete clinical and electrographic } \\
\text { response was obtained after KTM introduction; } \\
\text { after } 15 \text { days SE relapsed and the patient died. }\end{array}$ \\
\hline $\begin{array}{l}\text { Baxter } \\
\text { et al. } \\
{[83]}\end{array}$ & $\begin{array}{l}N=3 \\
\text { term }\end{array}$ & $\begin{array}{l}N=2 \text { EIEE } \\
N=1 \text { Aicardi- } \\
\text { Goutieres }\end{array}$ & $\begin{array}{l}\text { PHB } \\
\text { (dose: N/A) }\end{array}$ & $\begin{array}{l}\text { PYR, CLZ, VPA } \\
\text { VGB } \\
(40 \mathrm{mg} / \mathrm{kg} / \mathrm{d})\end{array}$ & $2 / 3$ patients showed full response to VGB. \\
\hline $\begin{array}{l}\text { Wolf et al. } \\
{[84]}\end{array}$ & $\begin{array}{l}N=1 \\
\text { term }\end{array}$ & $\begin{array}{l}\text { Incontinentia } \\
\text { pigmenti }\end{array}$ & $\begin{array}{l}\text { PHB } \\
(35 \mathrm{mg} / \mathrm{kg})\end{array}$ & $\begin{array}{l}\text { LRZ as } 2 \mathrm{nd} \text { line } \\
(0.2 \mathrm{mg} / \mathrm{kg}) \\
\text { PHE as } 3 \mathrm{rd} \text { line } \\
(20 \mathrm{mg} / \mathrm{kg}) \\
\text { Dexamethasone } \\
(0.25 \mathrm{mg} / \mathrm{kg} / \mathrm{d})\end{array}$ & $\begin{array}{l}\text { Rapid improvement and clinical seizures } \\
\text { termination followed the } \\
\text { initiation of CCS therapy. }\end{array}$ \\
\hline $\begin{array}{l}\text { Shevell } \\
\text { et al. } \\
{[85]}\end{array}$ & $\begin{array}{l}N=1 \\
\text { term }\end{array}$ & BFNE & $\begin{array}{l}\text { PHB } \\
(10 \mathrm{mg} / \mathrm{kg})\end{array}$ & / & $\begin{array}{l}\text { Patient presented no more seizures, was } \\
\text { discharged home on oral PHB, suspended at five } \\
\text { months of life }\end{array}$ \\
\hline $\begin{array}{l}\text { Lee et al. } \\
{[86]}\end{array}$ & $\begin{array}{l}N=1 \\
\text { term }\end{array}$ & KCNQ2 & $\begin{array}{l}\text { PHB } \\
(6 \mathrm{mg} / \mathrm{kg} / \text { day })\end{array}$ & $\begin{array}{l}\text { PHE as } 2 \mathrm{nd} \text { line } \\
(8 \mathrm{mg} / \mathrm{kg} / \text { day }) \\
\text { VGB } \\
(50 \mathrm{mg} / \mathrm{kg} / \text { day })\end{array}$ & $\begin{array}{l}\text { VGB reduced seizures; Once treatment with } \\
\text { Vigabatrin was administered seizures reduced to } \\
\text { one per day until day } 24 \text { of post-natal life, time } \\
\text { at which the last seizure was recorded. }\end{array}$ \\
\hline $\begin{array}{l}\text { Sato et al. } \\
{[87]}\end{array}$ & $\begin{array}{l}\mathrm{N}=2 \\
\text { late } \\
\text { preterm } \\
\text { and term }\end{array}$ & HIE & $\begin{array}{l}\text { PHB } \\
(10 \mathrm{mg} / \mathrm{kg})\end{array}$ & / & $\begin{array}{l}\text { Both patients temporarily controlled seizures on } \\
\text { PHB. One relapsed and developed severe DD. }\end{array}$ \\
\hline $\begin{array}{l}\text { Sillanpää } \\
\text { et al. } \\
\text { [88] }\end{array}$ & $\begin{array}{l}N=1 \\
\text { term }\end{array}$ & Feeding epilepsy & $\begin{array}{l}\text { PHB } \\
(60 \mathrm{mg} / \text { day }) \\
+ \\
\text { chlorpromazine } \\
\text { ( } 9 \mathrm{mg} / \text { day) }\end{array}$ & $\begin{array}{l}\text { The patient was seizure-free since } \\
\text { day } 14 \text { of PHB. }\end{array}$ & $\begin{array}{l}\text { Only few cases of neonatal feeding seizures are } \\
\text { described. In this case the patient was seizure- } \\
\text { free on PHB, after a six days combination- } \\
\text { therapy with chlorpromazine. }\end{array}$ \\
\hline $\begin{array}{l}\text { Tramonte } \\
\text { et al. } \\
\text { [89] }\end{array}$ & $\begin{array}{l}N=1 \\
\text { term }\end{array}$ & $\begin{array}{l}\text { Temporal lobe } \\
\text { hemorrhage }\end{array}$ & $\begin{array}{l}\text { PHB } \\
\text { (dose: N/A) }\end{array}$ & / & $\begin{array}{l}\text { After PHB's administration no more autonomic } \\
\text { seizures (apnea, desaturations) were noticed. }\end{array}$ \\
\hline
\end{tabular}

hospital stays. Of these 74 patients, 54 (73\%) patients responded to the administration of these drugs, gaining seizure control of the 54 patients that responded to treatment, 41 (76\% of patients that responded to treatment) were treated with carbamazepine, [61, 62, 72-74], $8(15 \%)$ were treated with phenytoin, $[61,71]$ and $4(7 \%)$ were treated with lidocaine [71, 75]. All 12 newborns treated with intravenous phenytoin and/or lidocaine were later transitioned to oral carbamazepine to maintain seizure control. Seizure control was maintained in 46 out of 54 patients $(85 \%)$ on oral carbamazepine (dose range: $10-30 \mathrm{mg} / \mathrm{kg} /$ day).

Based on the seizure type, onset, location, familial anamnesis, and EEG findings, diagnoses of BFNE were 
Table 5 Treatment outcomes in patients with hypoxic-ischemic encephalopathy

\begin{tabular}{|c|c|c|c|c|c|c|c|c|c|}
\hline & $\begin{array}{l}\mathrm{N} \text { of } \\
\text { patients } \\
\text { treated }\end{array}$ & $\begin{array}{l}\mathrm{N} \text { of } \\
\text { patients } \\
\text { treated as } \\
\text { 1st-line }\end{array}$ & $\begin{array}{l}\text { Efficacy as } 1 \text { st-line AED; } \\
\mathrm{N}(\%)\end{array}$ & $\begin{array}{l}\mathrm{N} \text { of patients } \\
\text { treated as } \\
\text { 2nd-line }\end{array}$ & $\begin{array}{l}\text { Efficacy } \\
\text { as 2nd- } \\
\text { line; } \\
\mathrm{N}(\%)\end{array}$ & $\begin{array}{l}\mathrm{N} \text { of patients } \\
\text { treated as } \\
\text { 3rd-line }\end{array}$ & $\begin{array}{l}\text { Efficacy } \\
\text { as 3rd- } \\
\text { line; } \\
\mathrm{N}(\%)\end{array}$ & $\begin{array}{l}\text { Overall } \\
\text { efficacy }\end{array}$ & Side-effects \\
\hline Phenobarbital & 76 & 76 & 49 (65\%) & / & I & I & I & $65 \%$ & None reported \\
\hline Lorazepam & 10 & 5 & $2(20 \%)$ & 5 & $2(20 \%)$ & & / & $4(40 \%)$ & $\begin{array}{l}\text { Liver enzyme } \\
\text { elevation in } 1 \\
\text { patient }\end{array}$ \\
\hline Midazolam & 333 & / & / & 226 & $14-50 \%$ & 107 & $57.5 \%$ & $36 \%$ & $\begin{array}{l}\text { Hypotension } \\
\text { in } 39 \text { patients } \\
(12 \%)\end{array}$ \\
\hline Lidocaine & 317 & / & / & 208 & $\begin{array}{l}94 \\
(45 \%)\end{array}$ & 125 & $\begin{array}{l}91 \\
(73.4 \%)\end{array}$ & $59 \%$ & None reported \\
\hline Levetiracetam & 76 & 22 & $\begin{array}{l}\text { Seizure freedom in } 11 \text { (50\%) } \\
\text { patients after } 40 \mathrm{~h} \text {, in } 22 \\
(100 \%) \text { after } 5 \text { days }\end{array}$ & 54 & $\begin{array}{l}49 \\
(92 \%)\end{array}$ & / & / & $75 \%$ & None reported \\
\hline Bumetanide & 14 & 14 & 7 (50\%) & / & / & / & / & N/A & Ototoxicity \\
\hline
\end{tabular}

made prior to genetic confirmation in 4 patients, who were treated with low-dose oral carbamazepine $(10 \mathrm{mg} /$ $\mathrm{kg}$ ), as a first-line anticonvulsant, and gained seizure freedom within hours of the first administration, with no need for further drug administration [62]. A long-term follow-up of these patients was provided, which demonstrated the maintenance of seizure-freedom for all of them. Among the 20 patients that did not respond, 15 were affected by KCNQ2 epileptic encephalopathy and responded to a combination of several drugs, including sodium channel blockers, topiramate, and levetiracetam $[43,60]$; Of the remaining patients, 3 were affected by KCNQ3 encephalopathy, and 2 of these patients dramatically responded to intravenous levetiracetam [43]. Another patient with a KCNT1 mutation was affected by refractory status epilepticus, and seizures decreased with intravenous levetiracetam [67]. One patient had refractory status epilepticus, due to SCN8A mutation [77]; unfortunately, he did not respond to a combination of drugs that included oxcarbazepine, phenobarbital, lamotrigine, and phenytoin.

Among our cohort, 2 patients were never treated with sodium channel blockers; the first was affected by BFNE, responded to phenobarbital, and treatment was slowly tapered until suspension within the first year of age [85]; however, due to the benign course of the condition, seizures may have stopped regardless of the treatment administered. The second patient, in contrast, was diagnosed with SCN1A mutation and was successfully treated with valproate after phenobarbital failure [86].

\section{Discussion}

The therapeutic management of seizures in the newborns has remained unchanged for decades, despite almost 20 years evidence that commonly-used medications are not only ineffective but also potentially neurotoxic for newborns.

This systematic review aimed to collect all of the available data from existing studies published in the literature that have examined the currently available pharmacological treatments of electrically-confirmed neonatal seizures, describing the real-world effectiveness and side-effects associated with drug administration.

Our paper illustrates the limited available evidence regarding the best pharmacological treatments for neonatal seizures and serves as a reference for future studies.

International surveys among neonatologists, worldwide, have confirmed the historical trend toward the use of phenobarbital (in up to $70 \%$ of cases), as a first-line AED, and phenytoin (in up to $40 \%$ of cases), as a

Table 6 Treatment outcomes in patients with stroke

\begin{tabular}{llllll}
\hline $\begin{array}{l}\text { Population } \\
\end{array}$ & $\begin{array}{l}\text { First-line } \\
\text { AED }\end{array}$ & $\begin{array}{l}\text { Response to } \\
\text { first-line AED }\end{array}$ & Response to Lidocaine as a 2nd-/3rd-line AED & $\begin{array}{l}\text { Response to } \\
\text { Midazolam } \\
\text { as a 2nd-/3rd-line AED }\end{array}$ & $\begin{array}{l}\text { Response to other } \\
\text { AEDs }\end{array}$ \\
\hline $\begin{array}{lllll}\text { 45 } \\
\text { newborns } \\
\text { with stroke }\end{array}$ & $\begin{array}{l}45(100 \%) \\
\text { phenobarbital }\end{array}$ & $\begin{array}{l}4(5 \%) \text { did not } \\
\text { require further } \\
\text { treatment }\end{array}$ & $\begin{array}{l}\text { 27 out of 32 (84\%) patients treated with lidocaine } \\
\text { responded (higher in full-term newborns and } \\
\text { when used as a 2nd-line AED) }\end{array}$ & $\begin{array}{l}5 \text { out of 32 (16\%) } \\
\text { patients treated with } \\
\text { midazolam responded }\end{array}$ & $\begin{array}{l}1 \text { patient responded levetiracetam as a } \\
\text { 3rd-line AED }\end{array}$ \\
\hline
\end{tabular}


Table 7 Treatment outcomes of patients with early-onset epileptic encephalopathies

\begin{tabular}{|c|c|c|c|c|c|c|c|c|}
\hline $\begin{array}{l}\mathrm{N} \text { of } \\
\text { patients }\end{array}$ & $\begin{array}{l}\text { Gene } \\
\text { mutation }\end{array}$ & $\begin{array}{l}\text { Response to } \\
\text { carbamazepine } \\
\mathrm{N}(\%)\end{array}$ & $\begin{array}{l}\text { Response } \\
\text { to } \\
\text { lidocaine } \\
\mathrm{N}(\%)\end{array}$ & $\begin{array}{l}\text { Response } \\
\text { to } \\
\text { phenytoin } \\
\mathrm{N}(\%)\end{array}$ & $\begin{array}{l}\text { Response to } \\
\text { other AEDS } \\
\mathrm{N}(\%)\end{array}$ & Maintenance & Side effects & Follow-up \\
\hline 66 & KCNQ2 & $\begin{array}{l}40(61 \%) \\
\text { Carbamazepine }\end{array}$ & $\begin{array}{l}4(6 \%) \\
\text { lidocaine }\end{array}$ & $\begin{array}{l}7(10 \%) \\
\text { phenytoin }\end{array}$ & $\begin{array}{l}13(20 \%) \\
\text { combination of } \\
\text { drugs, including } \\
\text { Na-channel } \\
\text { blockers }\end{array}$ & $\begin{array}{l}44(66 \%) \\
\text { carbamazepine }\end{array}$ & $\begin{array}{l}1 \\
\text { methemoglobinemia } \\
\text { (on lidocaine) }\end{array}$ & $\begin{array}{l}\text { Follow-up: from } 3 \\
\text { months to } 10 \text { years. } \\
\text { Normal development } \\
\text { for BFNE; severe } \\
\text { developmental delay in } \\
\text { KCNQ2 encephalopathy }\end{array}$ \\
\hline 6 & KCNQ3 & $\begin{array}{l}1(16 \%) \\
\text { oxcarbazepine } \\
(20 \mathrm{mg} / \mathrm{kg})\end{array}$ & / & / & $\begin{array}{l}2(33 \%) \\
\text { levetiracetam } \\
(70-85 \mathrm{mg} / \mathrm{kg})\end{array}$ & $\begin{array}{l}1(16 \%) \\
\text { oxcarbazepine } \\
2(33 \%) \\
\text { levetiracetam }\end{array}$ & None reported & Normal up to 4 years \\
\hline 1 & KCNT1 & / & / & $0(0 \%)$ & $\begin{array}{l}\text { No response to } \\
\text { phenobarbital, } \\
\text { lamotrigine, or } \\
\text { benzodiazepines. } \\
1(100 \%) \\
\text { levetiracetam } \\
10-30 \mathrm{mg} / \mathrm{kg}\end{array}$ & $\begin{array}{l}\text { levetiracetam } \\
30 \mathrm{mg} / \mathrm{kg} / \text { day }\end{array}$ & None reported & $\begin{array}{l}\text { Seizure decrease (still } 1 \\
\text { episode/day) at } 14 \\
\text { months }\end{array}$ \\
\hline 1 & SCN2A & / & / & $\begin{array}{l}1(100 \%) \\
\text { phenytoin } \\
20 \mathrm{mg} / \mathrm{kg}\end{array}$ & / & $\begin{array}{l}\text { carbamazepine } \\
30 \mathrm{mg} / \mathrm{kg}\end{array}$ & None reported & $\begin{array}{l}\text { Severe developmental } \\
\text { delay at } 2 \text { years }\end{array}$ \\
\hline 1 & SCN1A & / & / & / & $\begin{array}{l}1(100 \%) \\
\text { valproate } 50 \\
\mathrm{mg} / \mathrm{kg}\end{array}$ & $\begin{array}{l}\text { valproate } 50 \\
\mathrm{mg} / \mathrm{kg}\end{array}$ & N/A & $\begin{array}{l}\text { Severe developmental } \\
\text { delay at } 3 \text { years }\end{array}$ \\
\hline 1 & SCN8A & $\begin{array}{l}0(0 \%) \\
\text { oxcarbazepine } \\
80 \mathrm{mg} / \mathrm{kg}\end{array}$ & & $\begin{array}{l}0(0 \%) \\
\text { phenytoin } \\
20 \mathrm{mg} / \mathrm{kg}\end{array}$ & $\begin{array}{l}\text { Seizure } \\
\text { reduction on a } \\
\text { combination of } \\
\text { oxcarbazepine, } \\
\text { phenytoin, and } \\
\text { lamotrigine }\end{array}$ & $\begin{array}{l}\text { phenytoin, } \\
\text { oxcarbazepine, } \\
\text { phenobarbital, } \\
\text { lamotrigine }\end{array}$ & N/A & $\begin{array}{l}\text { Daily seizures at } 6 \\
\text { months }\end{array}$ \\
\hline
\end{tabular}

second-line AED, regardless of the seizure etiology or gestational age $[90,91]$.

However, several studies have demonstrated that phenobarbital may have potential long-term side-effects on neurodevelopment, which is not often considered when making treatment decisions [92-96]. In addition, the overall efficacy of phenobarbital varies widely across reports, and in line with previous data from the literature, our review found that the overall efficacy of phenobarbital does not exceed $66 \%$ among all patients treated. Several preclinical studies have explored the poor efficacy of GABAergic drugs, such as phenobarbital and benzodiazepines, by demonstrating that inhibitory mechanisms are underdeveloped in the immature brain, in a manner that is directly proportional to gestational age [97-99]. Animal studies in P7 mice, a post-natal age that grossly corresponds with 30-32 weeks of human gestational age, have confirmed that GABA receptors and the enzymes involved in GABA synthesis are expressed at low levels at birth and increase with time, during the first weeks of life [100]. In particular, the poor efficacy of phenobarbital may represent a developmental consequence of the persistence of the immature form of the sodium-potassium- chloride transporter, NKCC1, which may compromise the chloride-concentration gradient that is essential to phenobarbital's mechanism of action [101]. Furthermore, GABA is known to act in an excitatory, rather than inhibitory [102], role during early stages of neurodevelopment, which may not only explain the ineffectiveness of GABA-enhancer drugs but also their potential roles during paradoxical seizure disruption.

Phenytoin and lidocaine appear to be potentially effective as second-line treatments for refractory seizures; however, to date, no strong evidence exists to recommend their use.

Phenytoin was shown to be effective in approximately $45 \%$ of patients during an RCT [23]. When added as a second-line treatment for seizures that were refractory to phenobarbital, phenytoin facilitated seizure control in an additional $10-15 \%$ of treated patients. Different studies have described higher risks of drug accumulation that reach toxic plasma concentrations when administered to preterm compared with full-term newborns. Because of its non-linear pharmacokinetic profile and hepatic metabolism, phenytoin administration also requires frequent blood-level monitoring, making it a slightly manageable medication. 
Overall, the effectiveness of lidocaine ranged from 20 to $81 \%$ of patients treated [24, 34, 52, 53, 55, 56]; among patients with HIE, however, we observed that only milder phenotypes responded well to lidocaine, whereas a much lower effectiveness rate $(30 \%)$ was reported for severely asphyxiated newborns [56]. Lidocaine, instead, appears to be more promising for the treatment of patients with stroke [52, 55], and among this population, functional studies demonstrated that lidocaine, in comparison with phenytoin, acted less strongly to suppress background activity and more strongly to suppressing electrical activity in specific ischemic areas of the brain. We observed that lidocaine administration in patients with stroke resulted in seizure control for $84 \%$ of patients treated, compared with much lower response rates for both midazolam and phenobarbital. In contrast, several other papers reported potential side-effects associated with lidocaine, including cardiac arrhythmias and hypotension. Therefore, lidocaine should not be used after phenytoin, due to the increased risk of cardiodepressive effects [103]. In addition, a seizure-inducing effect associated with high doses of lidocaine has been reported [104].

In the only RCT that compared midazolam and lidocaine for the treatment of neonatal seizures caused by various etiologies, a toward improved efficacy was observed for lidocaine, although both groups of patients had poor outcomes at 1 year of age [24]. Serious adverse side-effects, such as respiratory depression and sedation, have been reported and potential side-effects may also occur due to interactions between benzodiazepines and other pharmacological treatments. In addition, midazolam clearance correlates with gestational age, with reduced elimination observed among preterm infants, due to immature hepatic metabolism, which may result in a higher risk of side effects due to accumulation [105]. For these reasons, benzodiazepines should be considered second- or third-line treatments that are more suitable for already sedated and intubated newborns.

During the last few years, levetiracetam use has increased, due to the growing amount of literature regarding the safety and efficacy of both loading and maintenance doses and because several studies have reported that levetiracetam, in contrast with phenobarbital, is devoid of any pro-apoptotic properties that might affect the developing brain, even at exceptionally high doses $[106,107]$. In addition, both intravenous and enteral preparations are available, making levetiracetam extremely manageable for clinical use. Although the exact mechanism of action for levetiracetam remains unknown, it has been hypothesized to target the synaptic vesicle glycoprotein 2A (SV2A). Talos et al. [108] estimated that neonatal neuronal SV2A protein levels reach $94 \%$ of adult values by 37 weeks post-conceptional age, suggesting that the target for levetiracetam may be abundantly expressed, even in the immature neonatal brain.

We have observed that the efficacy of levetiracetam varies across studies, ranging from 32 to $100 \%$ of treated patients, for both full-term and preterm newborns [27$30,38-40,42,44-47,65]$. Stratifying patients by etiology allowed us to observe that, to date, more data regarding the efficacy of levetiracetam are available for patients with seizures due to HIE than those due to other causes, and in this population, levetiracetam was effective, providing seizure freedom in up to $50 \%$ of patients after 40 $\mathrm{h}$ of treatment, when used as a first-line, monotherapy, and in up to $92 \%$ of patients in a longer-term follow-up, as both a first- and second-line anticonvulsant $[46,47]$. In a population of 44 asphyxiated newborns, initial treatment with levetiracetam predicted a shorter interval to seizure freedom than treatment with phenobarbital in univariate analysis, even after adjusting for initial seizure frequency and unbiased HIE severity score [47]. In addition, comparison between levetiracetam and phenobarbital for the treatment of neonatal seizures caused by various etiologies showed a short-term better effect of levetiracetam on tone and posture of patients according to HNNE score [26]. Treatment doses ranged from 10 to $60 \mathrm{mg} / \mathrm{kg}$ for the loading dose, and from 10 to $80 \mathrm{mg} / \mathrm{kg}$ for the maintenance dose. No serious adverse events were reported associated with levetiracetam administration, except for mild somnolence and feeding difficulty, which were resolved by doseadjustment. Several studies focused on the safe and predictable pharmacokinetic profile of levetiracetam, even in preterm and extremely sick full-term newborns, emphasizing that because levetiracetam does not require hepatic metabolism, it rarely interferes with other treatments [109]. Considering its safety profile and higher distribution volume in newborns (0.89 compared with $0.6-0.7 \mathrm{~L} / \mathrm{kg}$ in children), we recommend the use of higher doses $(30-60 \mathrm{mg} / \mathrm{kg}$ for the loading dose and $30-50 \mathrm{mg} / \mathrm{kg} /$ day, divided into 2-3 doses for maintenance, eventually titrated up to $80 \mathrm{mg} / \mathrm{kg} /$ day) [110].

Several papers have described the efficacy of sodiumchannel blockers for the treatment of genetic channelopathies. Phenytoin, lidocaine, carbamazepine, and oxcarbazepine act to block the movement of sodium ions through ion channels during the propagation of action potentials to prevent seizure activity. Due to structural similarities, sodium channel blockers also act on potassium channels, resulting in seizure control in patients with genetic epilepsies due to KCNQ mutations. The modulation of one type of channel has also been hypothesized to affect the functions of the entire channel complex. 
In agreement with the literature, KCNQ2 mutations represented the most common genetic anomaly identified in our review. We observed a good response to treatment using sodium channel blockers in patients with these mutations, with an overall 63\% efficacy. A better response was observed for carbamazepine (77\% among responders to treatment), which was also the most commonly used medication because it has few to no reported side-effects and an oral, extremely manageable formulation is available [61, 62, 72-74]. A few patients were treated with lidocaine or phenytoin, who responded to drug administration, were later dismissed on oral carbamazepine for the maintenance of seizure freedom $[61,71,75,76]$.

Interestingly, based on clinical features, familial anamnesis, and EEG patterns, 4 patients were treated early with oral carbamazepine as a first-line anticonvulsant and responded with seizure cessation within hours after the initial first administration [62]. We also observed that the patients who did not respond to carbamazepine were those who displayed features of severe KCNQ2 encephalopathy. Some of these patients responded to combinations of medications that included sodium channel blockers.

Less is known about other genetic encephalopathies, such as the KCNT1-related epilepsy of infancy with migrating focal seizures. Quinidine may effectively block the pathogenic constitutive activation of the KCNT1 channel at the molecular level, but no data regarding its administration for the neonatal population are available, to date [111].

When a genetic channelopathy is suspected, based on clinical features, familial anamnesis, and EEG patterns, the response to treatment with sodium-channel blockers not only represents the best treatment option available but may also be an ex juvantibus criteria to obtain a diagnosis when waiting for genetic test results, encouraging their early use and administration.

A change to the current "one size fits all" treatment model, in which treatment protocols do not account for etiology as a factor, is necessary to accommodate the possibility of customized, patient-specific, precision medicine.

Unfortunately, current data do not yet allow current treatment protocols to be replaced because the populations described, worldwide, are too heterogeneous, both in terms of etiology and treatment. The evaluation of each drug's efficacy for the treatment of specific etiologies is difficult when the populations described include both preterm and full-term newborns with seizures caused by a variety of etiologies.

Current knowledge, however, allows us to highlight the good clinical and electrographic responses of genetic early-onset epilepsies to sodium channel blockers and the overall good response to levetiracetam, whose administration has also been demonstrated to be safe in both full-term and preterm newborns.

Future investigations should identify methods to better identify and distinguish, as early as possible, between acute seizures and neonatal-onset epilepsies, to facilitate patient-specific, minimally dangerous treatment options, which will offer newborns, especially preterm newborns, higher survival rates, better neurological outcomes, and a better long-term quality of life.

\section{Conclusions}

After more than 20 years of experience, limited evidence exists regarding the best pharmacologic treatments for neonatal seizures. Treatment, too often, remains guided by experience, because few RCTs have been performed and the data available from those that have been performed have not been significant.

Additional controlled trials and large prospective studies are urgently necessary to determine the correct drug choices, dosing regimens, and treatment durations for newborns that will result in better futures, in terms of both seizure freedom and neurocognitive outcome.

This systematic review of neonatal seizure treatment underlines the pitfalls in current neonatology practice and serves as a reference to guide future investigations.

\section{Abbreviations \\ RCT: Randomized clinical trial; EEG: Electroencephalography; aEEG: amplitude- integrated electroencephalography; AED: Antiepileptic drug; WHO: World Health Organization; HIE: Hypoxic-ischemic encephalopathy; IVH: Intraventricular hemorrhage; CNS: Central nervous system; IUGR: Intrauterine growth restriction; BFNE: Benign familial neonatal epilepsy; GABA: Gamma-aminobutyric acid; NKCC1: Sodium-potassium-chloride transporter; SV2A: Synaptic vesicle glycoprotein 2A; HNNE: Hammersmith Neonatal Neurological Examination}

\section{Acknowledgments}

We wish to thank Cambridge Proofreading LLC 828 W Grace Chicago, LL, 606013 USA Phone: (+ 1) 844-332- 5040 for editing this manuscript.

\section{Authors' contributions}

R. F., B.S., M.R., M.M., M.C., A.G., P.P. reviewed the literature, critically discussed various aspects of neonatal seizures treatment and read the manuscript; R.F., B.S., P.P. and A.G. wrote the manuscript and prepared tables and figures. The author(s) read and approved the final manuscript.

\section{Funding}

The authors did not receive any funding for the research.

\section{Availability of data and materials}

The datasets used and/or analyzed during the current study are available from the corresponding author on reasonable request.

\section{Declarations}

Ethics approvals and consent to participate Not applicable.

Consent for publication

Not applicable. 


\section{Competing interests}

The authors have no competing interests to declare.

\section{Author details \\ 'Neonatal Intensive Care Unit, A.O.U. San Marco-Policlinico, University of Catania, Via Carlo Azeglio Ciampi, 95121 Catania, Italy. ${ }^{2}$ Post graduate University of Catania, Catania, Italy. ${ }^{3}$ Unit of Clinical Pediatrics, A.O.U. "Policlinico", P.O. "G. Rodolico", University of Catania, Catania, Italy. Child Neuropsychiatry, A.O.U. San Marco- Policlinico, University of Catania, Catania, Italy. \\ Received: 16 December 2020 Accepted: 15 March 2021 \\ Published online: 07 April 2021} programme in Pediatrics, Department of Clinical and Experimental Medicine, ${ }^{4}$ Department of Clinical and Experimental Medicine Section of Pediatrics and

\section{References}

1. Scher MS. Neonatal seizure classification: a fetal perspective concerning childhood epilepsy. Epilepsy Res. 2006;70:S41-57.

2. Glass $\mathrm{H}$, Shellhaas RA, Wusthoff $\mathrm{CJ}$, et al. Contemporary profile of seizures in neonates: a prospective cohort study. J Pediatr. 2016;174:98-103.e1.

3. Scher MS, Aso K, Beggarly ME, Hamid MY, Steppe DA, Painter MJ. Electrographic seizures in preterm and full-term neonates: clinical correlates, associated brain lesions, and risk for neurologic sequelae. Pediatrics. 1993;91: 128-34.

4. Pisani F, Facini C, Pavlidis E, Spagnoli C, Boylan G. Epilepsy after neonatal seizures: Literature review. Eur J Paediatr Neurol. 2015;19(1):6-14.

5. Toet MC, Groenendaal F, Osredkar D, van Huffelen AC, de Vries LS. Postneonatal epilepsy following amplitudeintegrated EEG-detected neonatal seizures. Pediatr Neurol. 2005;32:241e7.

6. Donovan MD, Griffin BT, Kharoshankaya L, Cryan JF, Boylan GB. Pharmacotherapy for neonatal seizures: current knowledge and future perspectives. Drugs. 2016;76(6):647-61.

7. Tymofiyeva O, Hess CP, Xu D, Barkovich AJ. Structural MRI connectome in development: challenges of the changing brain. Br J Radiol. 2014;87: 20140086.

8. Glass HC, Wirrell E. Controversies in neonatal seizure management. J Child Neurol. 2009 May;24(5):591-9.

9. Soul JS. Acute symptomatic seizures in term neonates: Etiologies and treatments. Seminars in Fetal \& Neonatal Medicine. 2018;23(3):183-90.

10. Kang SK. Kadam. Neonatal Seizures: Impact on Neurodevelopmental Outcomes. Front. Pediatr. 2015;3:101.

11. Guillet R, Kwon J. Seizure recurrence and developmental disabilities after neonatal seizures: outcomes are unrelated to use of phenobarbital prophylaxis. J Child Neurol. 2007;22:389-95.

12. Akman O, Mosh SL, Galanopoulou AS. Early life status Epilepticus and stress have distinct and sex-specific effects on learning, subsequent seizure outcomes, including anticonvulsant response to phenobarbital. CNS Neurosci Ther. 2015;21:181-92.

13. Kim JS, Kondratyev A, Tomita Y, Gale K. Neurodevelopmental impact of antiepileptic drugs and seizures in the immature brain. Epilepsia. 2007:48: 19-26.

14. Pinchefsky EF, Hahn CD. Outcomes following electrographic seizures and electrographic status epilepticus in the pediatric and neonatal ICUs. Curr Opin Neurol. 2017:30:156-64.

15. World Health Organization, Guidelines on neonatal seizures, 2011

16. Bittigau P, Sifringer M, Genz K, et al. Antiepileptic drugs and apoptotic neurodegeneration in the developing brain. Proc Natl Acad Sci U S A. 2002; 99:15089-94.

17. Ikonomidou C. Triggers of apoptosis in the immature brain. Brain and Development. 2009;31:488-92.

18. Soul JS, Pressler R, Allen M, et al. Recommendations for the design of therapeutic trials for neonatal seizures. Pediatr Res. 2019;85(7):943-54.

19. Wassinik G, Davidson JO, Lear CA, et al. A working model for hypothermic neuroprotection. J Physiol. 2018;596(23):5641-54.

20. Moher D, Shamseer L, Clarke M, Ghersi D, Liberati A, Petticrew M, Shekelle $P$, Lesley A Stewart and PRISMA-P Group. Preferred reporting items for systematic reviewand meta-analysis protocols (PRISMA-P) 2015 Statement. Syst Rev. 2015;4(1):1.

21. Contributors Institute of Medicine (US) Committee on Standards for Systematic Reviews of Comparative Effectiveness Research; Jill Eden, Laura
Levit, Alfred Berg, and Sally Morton. Finding What Works in Health Care. Standards for Systematic Reviews. 2011.

22. Shea BJ, Reeves BC, Wells G, Thuku M, Hamel C, Moran J, Moher D, Tugwell P. Welch V, Kristjansson E, Henry DA. AMSTAR 2: a critical appraisal tool for systematic reviews that include randomised or non-randomised studies of healthcare interventions, or both. BMJ. 2017;358:14008.

23. Painter MJ, Scher MS, Stein AD, et al. Phenobarbital compared with phenytoin for the treatment of neonatal seizures. N Engl J Med. 1999;341: 485-9.

24. Boylan G, Rennie J, Chorley G, et al. Second-line anticonvulsant treatment of neonatal seizures. Neurology. 2004;62:486-8.

25. Pressler RM, Boylan GB, Marlow N, et al. Bumetanide for the treatment of seizures in newborn babies with hypoxic ischaemic encephalopathy (NEMO): an open-label, dose finding, and feasibility phase 1/2 trial. Lancet Neurol. 2015:14:469-77.

26. Falsaperla R, Mauceri L, Pavone P, Barbagallo M, Vitaliti G, Ruggieri M, Pisani F, Corsello G. Short-Term Neurodevelopmental Outcome in Term Neonates Treated with Phenobarbital versus Levetiracetam: A Single-Center Experience. Behav Neurol. 2019;2019:3683548.

27. Ramantani G, Ikonomidou C, Walter B, Rating D, Dinger J. Levetiracetam: safety and efficacy in neonatal seizures. Eur J Pediatr Neurol. 2011;15:1-7.

28. Sharpe CM, Capparelli EV, Mower A, Farrell MJ, Soldin SJ, Haas RH. A sevenday study of the pharmacokinetics of intravenous levetiracetam in neonates: marked changes in pharmacokinetics occur during the first week of life. Pediatr Res. 2012;72(1):43-9.

29. Fürwentsches A, Cornelia Bussmann C, Georgia Ramantani G, et al. Levetiracetam in the treatment of neonatal seizures: a pilot study. Seizure. 2010;19(3):185-9.

30. Falsaperla R, Vitaliti G, Mauceri L, et al. Levetiracetam in neonatal seizures as first-line treatment: a prospective study. J Pediatr Neurosci. 2017:12(1):24-8.

31. Boylan GB, Pressler RM, Rennie JM, et al. Outcome of electroclinical, electrographic, and clinical seizures in the newborn infant. Dev Med Child Neurol. 1999;41:819-25.

32. Low E, Stevenson NJ, Mathieson SR, et al. Short-term effects of Phenobarbitone on electrographic seizures in neonates. Neonatology. 2016; 110(1):40-6.

33. Van der Broek MP, Van Straaten H, Huitema A, et al. Anticonvulsant effectiveness and hemodynamic safety of midazolam in full-term infants treated with hypothermia. Neonatology. 2015;107:150-6.

34. Hellstrom-Westas L, Svenningsen NW, Westgren U, Rostn I. Lagerstrom P; Lidocaine for treatment of severe seizures in newborn infants. Blood concentrations of lidocaine and metabolites during intravenous infusion. Acta Paediatr. 1992:81:35-9.

35. Maytal J, Novak GP, King KC. Lorazepam in the treatment of refractory neonatal seizures. J Child Neurol. 1991:6(4):319-23.

36. Glass $H$, Shellhaas $R$, Tsuchida $T$, et al. Seizures in preterm neonates: multicenter observational cohort study. Pediatr Neurol. 2017:72:19-24.

37. Glass HC, Soul JS, Chu CJ, et al. Response to antiseizure medications in neonates with acute symptomatic seizures. Epilepsia. 2019;60(3): e20-4

38. Abend NS, Gutierrez-Colina AM, Monk HM, et al. Levetiracetam for treatment of neonatal seizures. J Child Neurol. 2011;26:465-70.

39. Khan O, Chang E, Cipriani C, et al. Use of intravenous levetiracetam for management of acute seizures in neonates. Pediatr Neurol. 2011;44: 265-9.

40. Khan O, Cipriani C, Wright Pharm C, Crisp E, Kirmani B. Role of intravenous Levetiracetam for acute seizure Management in Preterm Neonates. Pediatr Neurol. 2013:49:340-3.

41. Rakshasbhuvankar A, Rao S, Kohan R, Simmer K, Nagarajan L, et al. Intravenous levetiracetam for treatment of neonatal seizures. J Clin Neurosci. 2013;20(8):1165-7.

42. Lo-Yee Yau M, Lai-Wah Fung E, Cheung NP. Response of levetiracetam in neonatal seizures. World J Clin Pediatr. 2015;4(3):45-9.

43. Maljevic S, Vejzovic S, Bernhard MK, et al. Novel KCNQ3 mutation in a large family with benign familial neonatal epilepsy: a rare cause of neonatal seizures. Mol Syndromol. 2016;7:189-96.

44. Shin JW, Jung YS, Park K, et al. Experience and pharmacokinetics of Levetiracetam in Korean neonates with neonatal seizures. Korean J Pediatr. 2017:60(2):50-4

45. Han JY, Moon CJ, Youn YA, Sung IK, Lee IG. Efficacy of levetiracetam for neonatal seizures in preterm infants. BMC Pediatr. 2018;18(1):131. 
46. Venkatesan C, Young S, Schapiro M, Thomas C. Levetiracetam for the treatment of seizures in neonatal hypoxic ischemic encephalopathy. J Child Neurol. 2017;32(2):210-4.

47. Rao LM, Hussain SA, Zaki T, et al. A comparison of levetiracetam and phenobarbital for the treatment of neonatal seizures associated with hypoxic-ischemic encephalopathy. Epilepsy Behav. 2018;88:212-7.

48. Van der Broek MPH, Groenendaal F, Toet MC, et al. Pharmacokinetics and clinical efficacy of phenobarbital in asphyxiated newborns treated with hypothermia. A Thermopharmacological Approach. Clin Pharmacokinet. 2012:51:671-9.

49. Boylan G, Rennie J, Pressler R, et al. Phenobarbitone, neonatal seizures, and video-EEG. Arch Dis Child Fetal Neonatal Ed. 2002;86:F165-70.

50. Spagnoli C, Seri S, Pavlidis E, Mazzotta S, Pelosi A, Pisani F. Phenobarbital for neonatal seizures: response rate and predictors of refractoriness. Neuropediatrics. 2016;47(5):318-26.

51. Hakeem VF, Wallace SJ. EEG monitoring of therapy for neonatal seizures. Dev Med Child Neurol. 1990:32:858-64

52. Weeke LC, Toet MC, Van Rooij LGM, et al. Lidocaine response rate in aEEGconfirmed neonatal seizures: Retrospective study of 413 full-term and preterm infants. Epilepsia. 2016;57(2):233-42.

53. Lundqvist M, Agren J, Hellstrom-Westas L, Flink R, Wickstrom R. Efficacy and safety of lidocaine for treatment of neonatal seizures. Acta Paediatr. 2013; 102(9):863-7.

54. Van der Broek MPH, Rademaker CMA, van Straaten HLM, et al. Anticonvulsant treatment of asphyxiated newborns under hypothermia with lidocaine: efficacy, safety and dosing. Arch Dis Child Fetal Neonatal. 2013;98:F341-5.

55. Jennekens W, Dankers $F$, Janssen $F$, et al. Effects of midazolam and lidocaine on spectral properties of the EEG in full-term neonates with stroke. Eur Paediatr Neurol. 2012;16(6):642-52.

56. Shany E, Oshra Benzaqen O, Watemberg N. Comparison of continuous drip of midazolam or Lidocaine in the treatment of intractable neonatal seizures. J Child Neurol. 2007;22(3):255-9.

57. McDermott CA, Kowalczyk AL, Schnitzler ER, Mangurten HH, Rodvold KA, Metrick S. Pharmacokinetics of Lorazepam in critically ill neonates with seizures. J Pediatr. 1992;120(3):479-83.

58. Castro-Conde JR, Borges AAH, Martinez ED, et al. Midazolam in neonatal seizures with no response to phenobarbital. Neurology. 2005;64:876-9.

59. Vilan A, Mendes Ribeiro J, Striano P, et al. A distinctive Ictal amplitudeintegrated electroencephalography pattern in newborns with neonatal epilepsy associated with KCNQ2 mutations. Neonatology. 2017:112:387-93.

60. Hortigüela M, Fernández-Marmiesse A, Cantarín V, et al. Clinical and genetic features of 13 Spanish patients with KCNQ2 mutations. J Hum Genet. 2017 Feb;62(2):185-9.

61. Pisano $T$, Numis $A L$, Heavin $S B$, et al. Early and effective treatment of KCNQ2 encephalopathy. Epilepsia. 2015;56(5):685-91.

62. Sands TT, Balestri M, Bellini $G$, et al. Rapid and safe response to low-dose carbamazepine in neonatal epilepsy. Epilepsia. 2016;57(12):2019-30.

63. Singh B, Singh P, Al Hifzi I, Khan M, Majeed-Saidan M. Treatment of neonatal seizures with carbamazepine. J Child Neurol. 1996;11:378-82.

64. Glass HC, Poulin C, Shevell MI. Topiramate for the treatment of neonatal seizures. Pediatr Neurol. 2011;44:439-42.

65. Shoemaker MT, Rotenberg JS. Levetiracetam for the treatment of neonatal seizures. J Child Neurol. 2007;22:95-8.

66. Tanriverdi S, Terek D, Koroglu OA, Yalaz M, Tekgul H, Kultursay N. Neonatal status epilepticus controlled with levetiracetam at Sturge weber syndrome. Brain and Development. 2013;35(4):367-71.

67. Hmaimess G, Kadhim H, Nassogne MC. Christine bonnier, Van Rijckevorsel K. Levetiracetam in a neonate with malignant migrating partial seizures. Pediatr Neurol. 2006;34(1):55-9.

68. Ledet DS, Wheless JS, Rubnitz JE, Morris B. Levetiracetam as Monotherapy for seizures in a neonate with acute lymphoblastic leukemia. Eur J Paediatr Neurol. 2010;14(1):78-9.

69. Li T, Cheng M, Wang J, et al. De novo mutations of STXBP1in Chinese children with early onset epileptic encephalopathy. Genes Brain Behav. 2018;17(8):e12492.

70. Dilena R, Striano P, Gennaro E, et al. Efficacy of sodium channel blockers in SCN2A early infantile epileptic encephalopathy. Brain and Development. 2017:39(4):345-8.
71. Bohnhorst $B$, Hartmann $H$, Lange M. Severe methemoglobinemia caused by continuous lidocaine infusion in a term neonate. Eur J Paediatr Neurol. 2017;21(3):576-9.

72. Numis AL, Angriman M, Sullivan JE, et al. KCNQ2 encephalopathy: delineation of the electroclinical phenotype and treatment response. Neurology. 2014:82(4):368-70.

73. Spagnoli C, Salerno GG, lodice A, Frattini D, Pisani F, Fusco C. KCNQ2 encephalopathy: A case due to a de novo deletion. Brain and Development. 2018;40(1):65-8.

74. Blumkin L, Suls A, Deconinck T, et al. Neonatal seizures associated with a severe neonatal myoclonus like dyskinesia due to a familial KCNQ2 gene mutation. Eur J Paediatr Neurol. 2012;16(4):356-60.

75. Buttle SG, Sell E, Dyment D, Bulusu S, Pohl D. Pointed rhythmic theta waves: a unique EEG pattern in KCNQ2-related neonatal epileptic encephalopathy. Epileptic Disord. 2017;19(3):351-6.

76. Soldovieri MV, Ambrosino P, Mosca I, Miceli F, Franco C, Canzoniero LMT, Kline-Fath B, Cooper EC, Venkatesan C, Taglialatela M. Epileptic encephalopathy in a patient with a novel variant in the Kv7.2 S2 Transmembrane segment: clinical, genetic, and functional feature. Int J Mol Sci. 2019;14:3382.

77. McNally MA, Johnson J, Huisman TA, et al. SCN8A epileptic encephalopathy: detection of fetal seizures guides multidisciplinary approach to diagnosis and treatment. Pediatr Neurol. 2016;64:87-91.

78. Okumura A, Yamamoto T, Shimojima K, et al. Refractory neonatal epilepsy with a de novo duplication of chromosome 2q24.2q24.3. Epilepsia. 2011; 52(7):e66-9.

79. Riesgo R, Winckler Ml, Ohlweiler $L$, et al. Treatment of refractory neonatal seizures with Topiramate. Neuropediatrics. 2012:43:353-6.

80. Sirsi D, Nangia S, LaMothe J, Kosofsky BE, Solomon GE. Successful management of refractory neonatal seizures with midazolam. J Child Neurol. 2008;23:706-9.

81. Steinberg A, Shalev RS, Amir N. Valproic acid in neonatal status convulsivus. Brain and Development. 1986;8:278-80.

82. Tarocco A, Ballardini E, Garani G. Use of ketamine in a newborn with refractory status Epilepticus: a case report. Pediatr Neurol. 2014;51(1):154-6.

83. Baxter PS, Gardner-Medwin D, Barwick DD, Incet P, Livingston J, MurdochEaton D. Vigabatrin monotherapy in resistant neonatal seizures. Seizure. 1995:4:57-9.

84. Wolf DS, Christopher Golden W, Hoover-Fong J, et al. High-dose glucocorticoid therapy in the Management of Seizures in neonatal Incontinentia Pigmenti: a case report. J Child Neurol. 2015;30(1):100-6.

85. Shevell MI, Sinclair DB, Metrakos K. Benign familial neonatal seizures: clinical and electroencephalographic characteristics. Pediatr Neurol. 1986;2:272-5.

86. Lee I-C, Chen J-Y, Chen Y-J, Yu J-S, Su P-H. Benign familial neonatal convulsions: novel mutation in a newborn. Pediatr Neurol. 2009:40:387-91.

87. Sato Y, Okumura A, Kato T, Hayakawa F, Kuno K, Watanabe K. Hypoxic ischemic encephalopathy associated with neonatal seizures without other neurological abnormalities. Brain and Development. 2003;25(3):215-9.

88. Sillanpää M, Schmidt D. Neonatal eating epilepsy: 50-year follow-up. Seizure. 2014;23(6):487-9.

89. Tramonte JJ, Goodkin HP. Temporal lobe hemorrhage in the full-term neonate presenting as apneic seizures. J Perinatol. 2004;24:726-9.

90. Glass HC, Kan J, Bonifacio SL, Ferriero DM. Neonatal seizures: treatment practices among term and preterm infants. Pediatr Neurol. 2012;46:111-5.

91. Dilena R, De Liso P, Di Capua M, et al. Influence of etiology on treatment choices for neonatal seizures: a survey among pediatric neurologists. Brain and Development. 2019;41(7):595-9.

92. Bhardwaj SK, Forcelli PA, Palchik G, Gale K, Srivastava LK, Kondratyev A. Neonatal exposure to phenobarbital potentiates schizophrenialike behavioral outcomes in the rat. Neuropharmacology. 2012;62(7):2336-44.

93. Forcelli PA, Janssen MG, Vicini S, Gale K. Neonatal exposure to antiepileptic drugs disrupts striatal synaptic development. Ann Neurol. 2012;72(3):363-72.

94. Torolira D, Suchomelova L, Wasterlain CG, Niquet J. Phenobarbital and midazolam increase neonatal seizure-associated neuronal injury. Ann Neurol. 2017:82(1):115-20.

95. Maartens IA, Wassenberg T, Jan Buijs J, Levinus Bok L, De Kleine MJK, Katgert T, Andriessen P. Neurodevelopmental outcome in full-term newborns with refractoryneonatal seizures. Acta Paediatr. 2012;101:e173-8.

96. Dessens AB, Cohen-Kettenis PT, Mellenbergh GJ, Koppe JG, van De Poll NE, Boer K. Association of prenatal phenobarbital and phenytoin exposure with 
small head size at birth and with learning problems. Acta Paediatr. 2000;89: 533-41.

97. Mallard C, Vexler ZS. Modeling ischemia in the immature brain how translational are animal models? Stroke. 2015;46(10):3006-11.

98. Katsarou AM, Galanopoulou AS, Moshé SL. Epileptogenesis in neonatal brain. Semin Fetal Neonatal Med. 2018;23(3):159-67.

99. Carrasco M, Stafstrom CE. How early can a seizure happen? Pathophysiological considerations of extremely premature infant. Dev Neurosci. 2018;40(5-6):417-36.

100. Brooks-Kayal AR, Shumate MD, Jin H, Rikhter TY, Kelly ME, Coulter DA. Gamma-Aminobutyric acid(a) receptor subunit expression predicts functional changes in hippocampal dentate granule cells during postnatal development. J Neurochem. 2001;77:1266-78.

101. Dzhala VI, Talos DM, Sdrulla DA, et al. NKCC1 transporter facilitates seizures in the developing brain. Nat Med. 2005:11:1205-13.

102. Cherubini E, Gaiarsa JL, Ben-Ari Y. GABA: an excitatory transmitter in early postnatal life. Trends Neurosci. 1991;14:515-9.

103. Wood RA. Sinoatrial arrest: an interaction between phenytoin and lignocaine. Br Med J. 1971;1:645.

104. Malingré MM, Van Rooij LG, Rademaker CM, et al. Development of an optimal lidocaine infusion strategy for neonatal seizures. Eur J Pediatr. 2006; 165:598-604.

105. Jacqz-Aigrain E, Daoud P, Burtin P, Maherzi S, Beaufils F. Pharmacokinetics of midazolam during continuous infusion in critically ill neonates. Eur J Clin Pharmacol. 1992;42:329-32.

106. Manthey D, Asimiadou S, Stefovska V, et al. Sulthiame but not levetiracetam exerts neurotoxic effect in the developing rat brain. Exp Neurol. 2005;193: 497-503.

107. Kim J-S, Kondratyev A, Tomita Y, Gale K. Neurodevelopmental impact of antiepileptic drugs and seizures in the immature brain. Epilepsia. 2007;48(5): 19-26.

108. Talos DM, Chang M, Kosaras B, et al. Antiepileptic effects of levetiracetam in a rodent neonatal seizure model. Pediatr Res. 2013;73:24-30.

109. Agrawal A, Banergee A. A review on pharmacokinetics of Levetiracetam in neonates. Curr Drug Metab. 2017 Oct 16;18(8):727-34.

110. Tulloch JK, Carr RR, Ensom MH. A systematic review of the pharmacokinetics of antiepileptic drugs in neonates with refractory seizures. J Pediatr Pharmacol Ther. 2012;17:31-44.

111. Numis AL, Umesh Nair U, Datta AN, et al. Lack of response to quinidine in KCNT1-related neonatal epilepsy. Epilepsia. 2018 Oct;59(10):1889-98.

\section{Publisher's Note}

Springer Nature remains neutral with regard to jurisdictional claims in published maps and institutional affiliations.

Ready to submit your research? Choose BMC and benefit from:

- fast, convenient online submission

- thorough peer review by experienced researchers in your field

- rapid publication on acceptance

- support for research data, including large and complex data types

- gold Open Access which fosters wider collaboration and increased citations

- maximum visibility for your research: over $100 \mathrm{M}$ website views per year

At $\mathrm{BMC}$, research is always in progress.

Learn more biomedcentral.com/submissions 